\title{
Design and Validation of a Novel Cable-Driven Hyper-Redundant Robot Based on Decoupled Joints
}

\author{
Long Huang, ${ }^{1,2}$ Bei Liu $\left(D,{ }^{1}\right.$ Lairong Yin $\left(D,{ }^{1}\right.$ Peng Zeng, ${ }^{1}$ and Yuanhan Yang ${ }^{1}$ \\ ${ }^{1}$ School of Automotive and Mechanical Engineering, Changsha University of Science and Technology, Changsha 410114, China \\ ${ }^{2}$ Hunan Provincial Key Laboratory of Intelligent Manufacturing Technology for High-performance Mechanical Equipment, \\ Changsha University of Science and Technology, Changsha 410114, China
}

Correspondence should be addressed to Bei Liu; 17871947856@163.com and Lairong Yin; yinlairong@csust.edu.cn

Received 15 July 2021; Revised 26 August 2021; Accepted 9 September 2021; Published 2 November 2021

Academic Editor: Yaoyao Wang

Copyright (c) 2021 Long Huang et al. This is an open access article distributed under the Creative Commons Attribution License, which permits unrestricted use, distribution, and reproduction in any medium, provided the original work is properly cited.

In most of the prior designs of conventional cable-driven hyper-redundant robots, the multiple degree-of-freedom (DOF) bending motion usually has bending coupling effects. It means that the rotation output of each DOF is controlled by multiple pairs of cable inputs. The bending coupling effect will increase the complexity of the driving mechanism and the risk of slack in the driving cables. To address these problems, a novel 2-DOF decoupled joint is proposed by adjusting the axes distribution of the universal joints. Based on the decoupled joint, a 4-DOF hyper-redundant robot with two segments is developed. The kinematic model of the robot is established, and the workspace is analyzed. To simplify the driving mechanism, a kinematic fitting approach is presented for both proximal and distal segments and the mapping between the actuator space and the joint space is significantly simplified. It also leads to the simplification of the driving mechanism and the control system. Furthermore, the cable-driven hyper-redundant robot prototype with multiple decoupled joints is established. The experiments on the robot prototype verify the advantages of the design.

\section{Introduction}

In recent years, due to the advantages of compact structure and flexible bending motion in the confined environment, hyper-redundant robots have received high attention in the field of minimally invasive surgery, maintenance, and testing [1-3]. Various kinds of cable-driven hyper-redundant robots have been reported by researchers [4-11]. Generally, the cable-driven hyper-redundant robots are usually composed of several segments driven by external actuators and multiple cables. Every segment consists of several identical joints in serial. Joint types for the cable-driven hyper-redundant robot mainly can be classified into the 1-DOF joint and 2DOF joint [12]. The 1-DOF joints mainly include the revolute joint $[13,14]$, the flexible beam $[15,16]$, and the cylindrical rolling joint $[17,18]$, while the multi-DOF joints mainly include the universal joint $[19,20]$, the flexible backbone [21, 22], and the spherical rolling joint. Based on the flexible backbone, Li et al. developed a 2-DOF flexible endoscope driven by multiple cables, which is more dexterous than rigid endoscopes [23]. Dong et al. proposed a continuum robot with a low ratio between diameter and length based on compliant joints, which can apply to the inspection and maintenance of aero engines [24]. Based on universal joints, Jin et al. designed cable-driven snake-like 4DOF surgical forceps [25]. Kim et al. designed a novel rolling joint with a block mechanism to develop a snake-like robot for minimally invasive surgery [26].

For most of the prior cable-driven hyper-redundant robots, the bending motion in all directions is usually coupled. It means the rotation output of each DOF is controlled by multiple pairs of cable inputs [27]. Besides, the mapping between the actuator space and joint space of the robot is complex, which causes the driving mechanism and the control system difficult [28]. Some researchers have tried to address the drawbacks of the coupling bending motion through special joint structure design and further simplify the mapping between actuator space and joint space $[29,30]$. 
Based on multiple cylindrical rolling joints, Kim et al. designed a cable-driven hyper-redundant robot, which diminishes the bending coupling effect by enlarging the space for the passage of the center cable [17].

In this paper, a novel 2-DOF decoupled joint is proposed by adjusting the distribution of two rotation axes. For the decoupled joint, a pair of antagonistic cable inputs only controls a 1-DOF rotational output, and the rotational output of each DOF is only determined by a single pair of antagonistic cable inputs. By connecting two 2-DOF segments in serial, a cable-driven hyper-redundant robot is presented. Each segment consists of multiple identical 2DOF decoupled joints in serial. A kinematics linear fitting approach is presented to simplify the mapping between actuator space and joint space. Based on the linear fitting error analysis, each pair of antagonistic cables is driven by a motor through a circular pulley, which can simplify the design of the driving mechanism. The proposed robot is verified by the bending motion experiments, the cable tension test, and the load experiments.

The rest of this paper is organized as follows: Section 2 introduces the challenges of the existing robots. Moreover, a novel cable-driven hyper-redundant robot is also introduced in Section 2. The kinematics and the robot workspace are analyzed in Section 3. The presented kinematics linear fitting and error analysis of the robot are discussed in Section 4. The robot prototype through some experiments is verified in Section 5. Section 6 presents the conclusion.

\section{Robot Design}

\subsection{Challenges of the Existing Robots}

2.1.1. Coupling Effect. For most of the prior designs of cabledriven hyper-redundant robots, the 2-DOF bending motion in each segment of the robot is coupled. The rotational output of each DOF of the joint is controlled by multiple pairs of antagonistic cable inputs. Consequently, the coupling effect will increase the complexity of the robot's driving mechanism and the risk of slack in the driving cables.

The relationship between the cable length and the rotation angles is determined by the joint types and their structural parameters. For instance, the coupling effect of the revolute joints is determined by the cable distribution circle radius, the number of cables, and the distribution of the rotation axes. Since the cable distribution circle radius and the number of cables are confined by the practical factors, the distribution of joint rotation axes is a significant factor to avoid the coupling effect. Figure 1 shows a conventional universal joint with two intersecting rotation axes located at the middle of two disks [31]. When the upper disk rotates around rotation axes $\mathbf{w}_{\mathbf{1}}$ through the releasing of cable $A$ and tightening of cable $C$, cable $B$ and cable $D$ must be tightened simultaneously to avoid slack, as shown in Figure 1(b). Similarly, the rotation around axis $\mathbf{w}_{2}$ requires the control of all four cables. Otherwise, cable $A$ and cable $B$ will become slack. It can be proved that the rotational output of each DOF requires the control of multiple pairs of antagonistic cables regardless of the location of the two intersecting axes in the joint. For the universal joint with two nonintersecting rotation axes located between the two disks [32], it can be also proved that the coupling effect exists in the joint when at least one rotation axis is located at the middle place of two disks, which is similar to the universal joint, as shown in Figure 1.

Based on the above analysis, we tried to change the distribution of the joint rotation axes to avoid the coupling effect. Therefore, this paper proposes a novel joint without coupling effects, as shown in Figure 2. The two axes in the joint are, respectively, coincident with the upper surface of the lower disk and the lower surface of the upper disk. Since points $A_{2}$ and $B_{2}$ are located at the axis $\mathbf{w}_{1}$, points $A_{2}$ and $B_{2}$ achieve circular motion around points $A_{1}$ and $B_{1}$ when the upper disk rotates around the axis $\mathbf{w}_{1}$. Therefore, the length of cable $A$ and cable $B$ will not change, while the length of cable $C$ and cable $D$ will also change. Since the points $C_{2}$ and $D_{2}$ are located at the axis $\mathbf{w}_{2}$, the points $C_{2}$ and $D_{2}$ can be considered as the fixed points when the upper disk rotates around the axis $\mathbf{w}_{2}$. In consequence, the length of cable $C$ and cable $D$ will not change, while the length of cable $A$ and cable $B$ will change. This indicates that the coupling effect does not exist in the proposed joint with the special distribution of two axes positions.

2.1.2. Challenge of the Driving Mechanism Design. The relationship between cable length and bending angles should be considered to design the driving mechanism of the cabledriven hyper-redundant robot [33, 34]. For most cabledriven hyper-redundant robots, the relationship between the length inputs of each cable and bending angles is a nonlinear function. It means the tightened amount on one cable is not equal to the released amount on the antagonistic cable when the robot bends to an arbitrary configuration. It is difficult for cable-driven hyper-redundant robots to design a compact and simple driving mechanism.

The following driving mechanism design approaches are adopted. The first approach is that each cable is driven by a separate motor and a circular cable pulley. It is convenient to design the driving mechanism. However, this approach increases the complexity of the control system, as shown in Figure 3(a). The second approach is that noncircular cable pulleys are designed to realize a motor driving a pair of cables based on the nonlinear function, as shown in Figure 3(b). This method significantly reduces the number of the motor, but the fabrication and assembly of noncircular cable pulleys require high accuracy. The third approach is that a pair of cables is driven by a separate motor and a circular cable pulley, as shown in Figure 3(c). This method requires the releasing amount of one cable is equal to the tightening amount of the antagonistic cable, which can simplify the driving mechanism.

2.2. A Novel Robot Design with the Decoupled Joints. This section proposes a cable-driven hyper-redundant robot based on the multiple decoupled joints, as shown in Figure 4. The robot is composed of a proximal segment, a distal segment, and a driving mechanism. Each segment consists of 


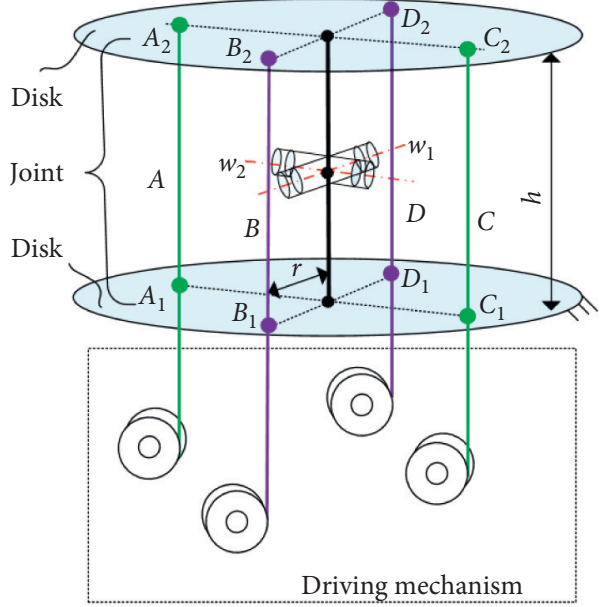

(a)

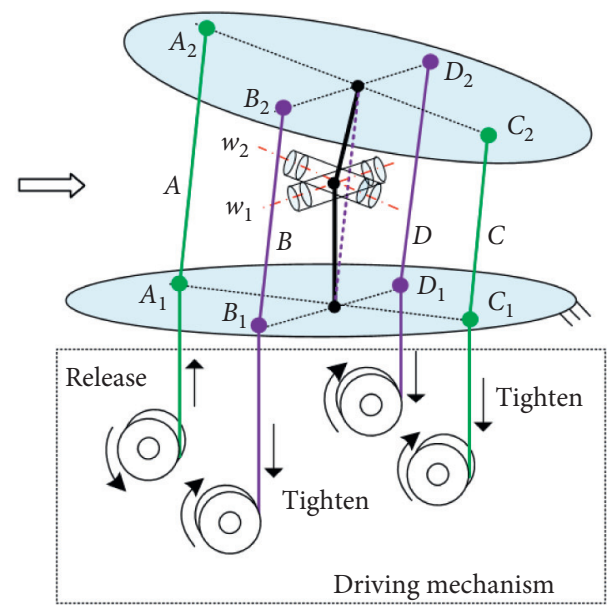

(b)

Figure 1: The traditional universal joint with two intersecting rotation axes.

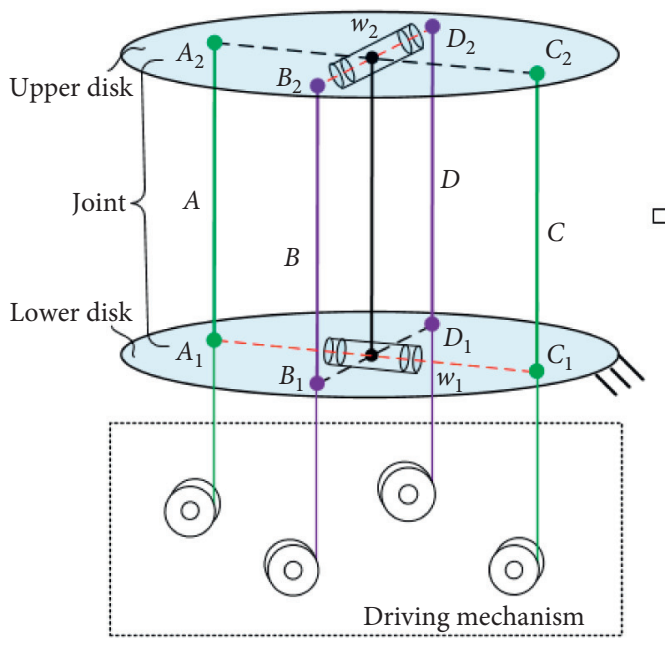

(a)

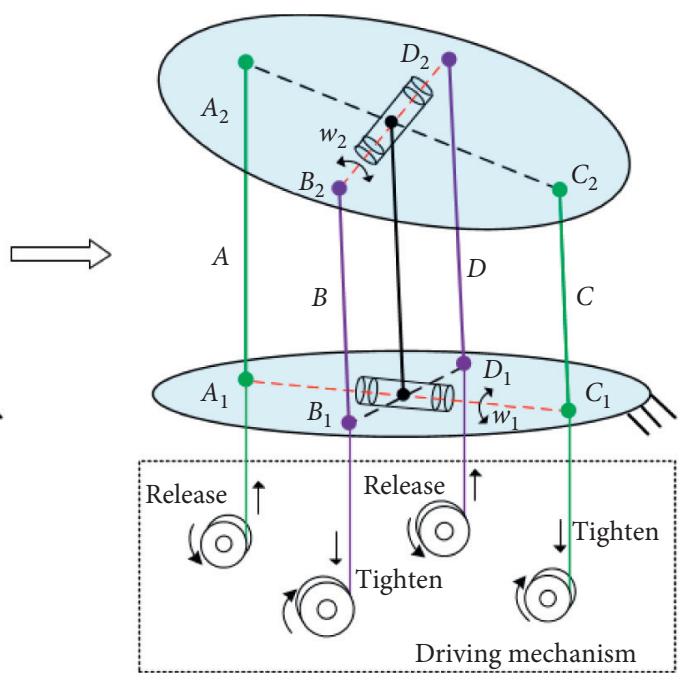

(b)

FIgURE 2: A novel joint without coupling effects.

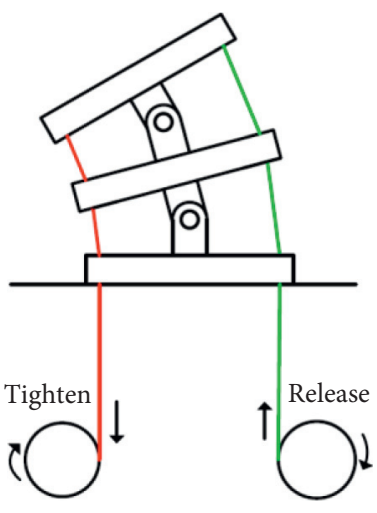

(a)

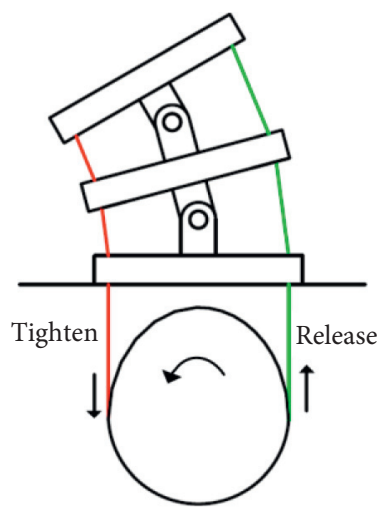

(b)

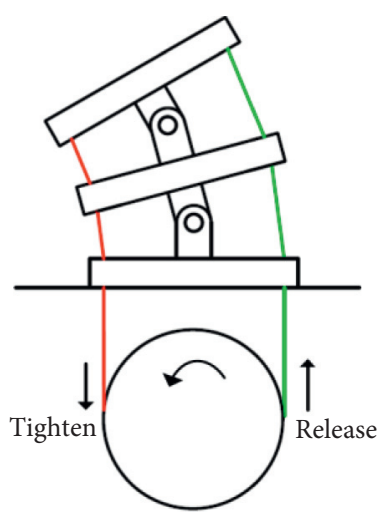

(c)

Figure 3: Classic driving system types. 


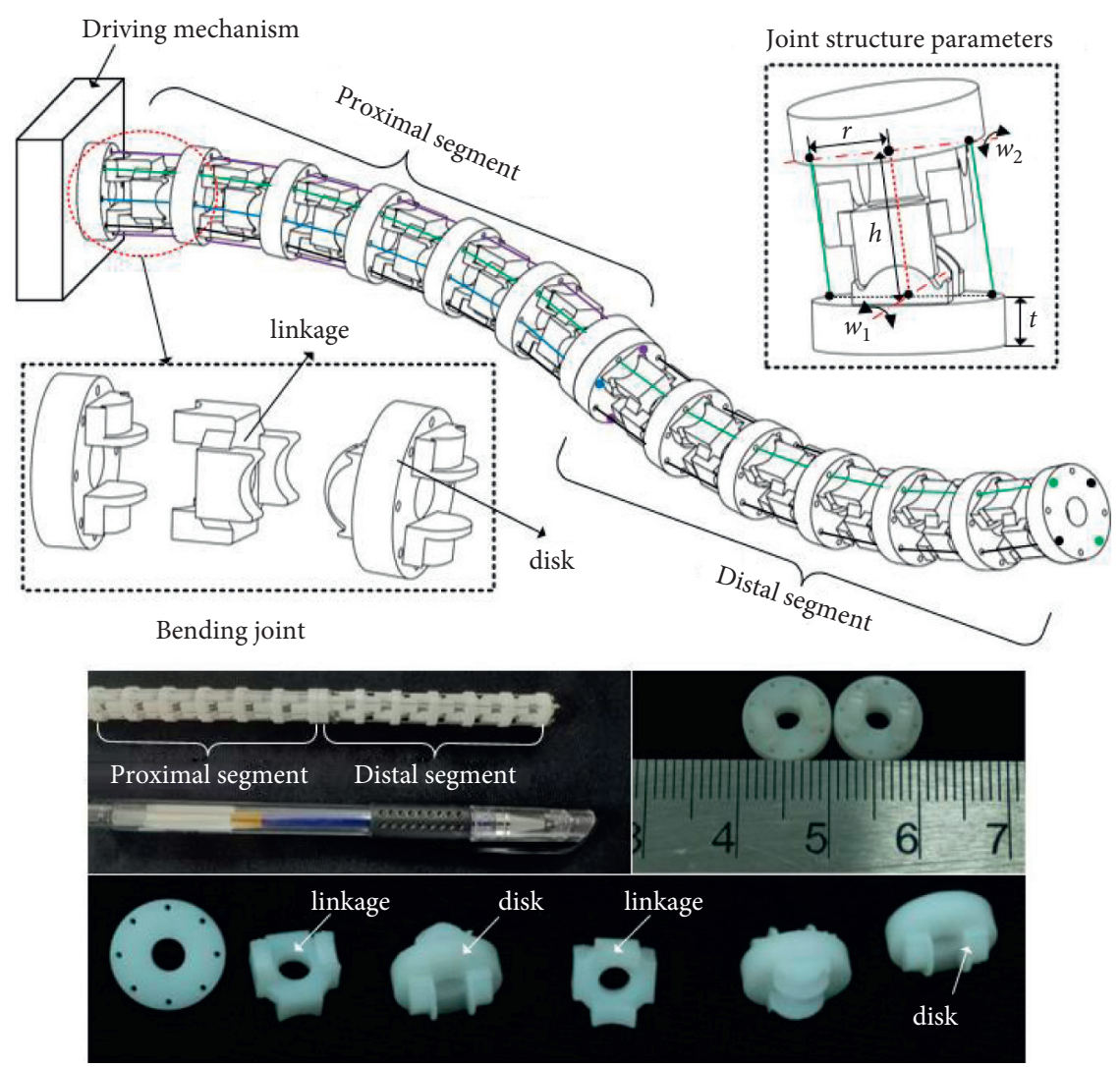

Figure 4: Cable-driven hyper-redundant robot prototype.

six identical 2-DOF joints. Each joint contains two disks and one spatial linkage. The cylindrical bulge surface on the disk and the cylindrical concave surface on the spatial linkage cooperate to form two rotating pairs. The axis $\mathbf{w}_{1}$ and axis $\mathbf{w}_{2}$ coincide, respectively, with the upper surface of the lower disk and the lower surface of the upper disk. Joint structure parameters are defined, as shown in Table 1.

The 2-DOF bending motion of each segment is achieved by two motors controlling a pair of antagonistic cables through the circular pulleys. Cable $A$, cable $B$, cable $C$, and cable $D$ control the 2-DOF bending motion of the proximal segment, as shown in Figure 5(a), while cable $E$, cable $F$, cable $G$, and cable $H$ control the 2-DOF bending motion of the distal segment, as shown in Figure 5(b). Since the two rotation axes of each joint are coincident with the end surfaces of corresponding disks, the distance of the two rotation axes is always equal to $h$ regardless of the robot configurations. In addition, a pair of antagonistic cable inputs only control the 1-DOF rotational output, and the rotational output of each DOF of the joint is only determined by a single pair of antagonistic cable inputs. With this design, the mapping between actuator space and joint space can be eventually simplified. Besides, the driving mechanism design of the robot is illustrated in Section 4.

\section{Kinematics}

The kinematics of the cable-driven hyper-redundant robot requires establishing the mapping between actuator space,
TABle 1: Parameters of the joint structure.

\begin{tabular}{lcc}
\hline Symbol & Description & Value \\
\hline$H$ & The distance between two axes & $8 \mathrm{~mm}$ \\
$t$ & Disk thickness & $2.5 \mathrm{~mm}$ \\
$r$ & Cable distribution circle radius & $4.25 \mathrm{~mm}$ \\
$d$ & Robot diameter & $10 \mathrm{~mm}$ \\
$(\theta, \varphi)$ & Joint variables in the proximal segment & $(-\pi / 18, \pi / 18)$ \\
$(\alpha, \beta)$ & Joint variables in the distal segment & $(-\pi / 18, \pi / 18)$ \\
\hline
\end{tabular}

joint space, and task space [35]. The following assumptions are made in this study. In this proposed robot, there is no gap between the cables and the cable holes. The cables' shear strains and elongation are negligible. The cable tension exerting on each joint is the same.

Based on these assumptions [36], the joint kinematics is first established to analyze the decoupled effect in the proposed 2-DOF joint. The relationship between the sum of the cable length change and bending angles theoretically validates that the cables in the robot will not become slack. Besides, the robot kinematics is established and the robot workspace is analyzed.

3.1. Joint Kinematics. Since the proximal segment and distal segment have the same bending motion, we consider a single joint in the proximal segment as an example to establish the joint kinematics, as shown in Figure 6.

The coordinate systems $\left\{\mathbf{O}_{i}\right\},\left\{\mathbf{O}_{1 i}\right\}$, and $\left\{\mathbf{O}_{i+1}\right\}$ are established, respectively, on the center of the upper surface 


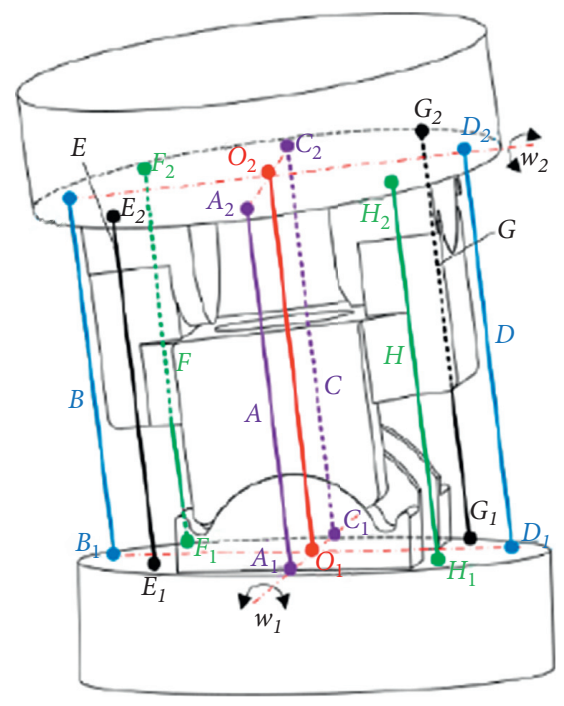

(a)

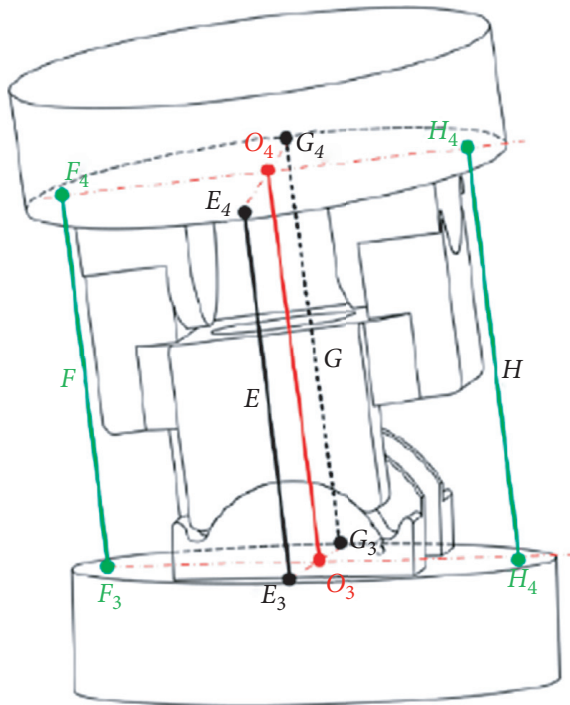

(b)

Figure 5: The 2-DOF joint of the proximal and distal segment.

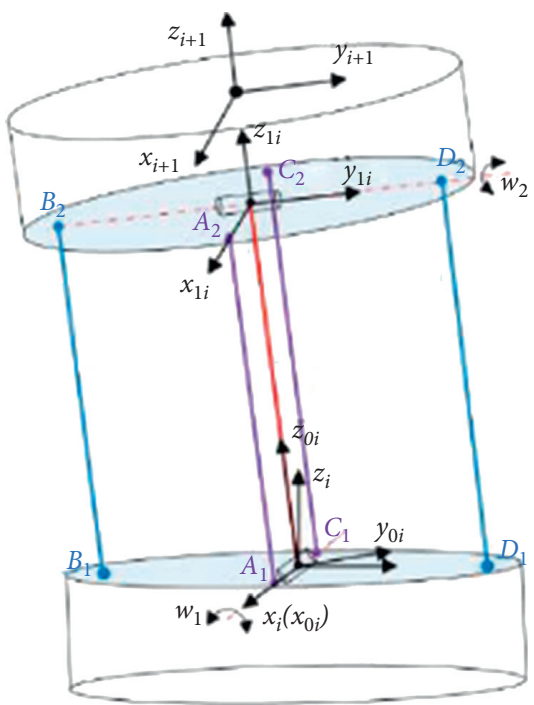

FIGURE 6: Kinematics coordinate system of the single joint.

of the lower disk, the lower surface of the upper disk, and the upper surface of the upper disk. Axis $\mathbf{x}_{i}$ coincides with the axis $\mathbf{w}_{1}$, axis $\mathbf{y}_{1 i}$ coincides with the axis $\mathbf{w}_{2}$, and axis $\mathbf{y}_{i+1}$ is parallel to the axis $\mathbf{w}_{2}$. The transformation from the coordinate system $\left\{\mathbf{O}_{i}\right\}$ to $\left\{\mathbf{O}_{i+1}\right\}$ is as follows. First, the coordinate system $\left\{\mathbf{O}_{i}\right\}$ rotates angle $\theta$ around $\boldsymbol{x}_{i}$ axis to obtain the coordinate system $\left\{\mathbf{O}_{0 i}\right\}$. Second, the coordinate system $\left\{\mathbf{O}_{0 i}\right\}$ moves $h$ along the $\mathbf{z}_{0 i}$ axis and then rotates angle $\varphi$ around the $\mathbf{y}_{0 i}$ axis to obtain the coordinate system $\left\{\mathbf{O}_{1 i}\right\}$. Third, the coordinate system $\left\{\mathbf{O}_{1 i}\right\}$ moves $t$ along the $\mathbf{z}_{1 i}$ axis to obtain the coordinate system $\left\{\mathbf{O}_{i+1}\right\}$. Hence, the homogeneous transformation matrix from the coordinate system $\left\{\mathbf{O}_{i}\right\}$ to $\left\{\mathbf{O}_{i+1}\right\}$ can be obtained as

$$
{ }_{i+1}^{i} \mathbf{T}=\operatorname{rot}\left(x_{i}, \theta\right) \operatorname{trans}\left(z_{0 i}, h\right) \operatorname{rot}\left(y_{0 i}, \varphi\right) \operatorname{trans}\left(z_{1 i}, t\right) \text {. }
$$

To establish the relationship between the cable length and bending angles, it is assumed that the position vector of any point $p_{1}$ in $\left\{\mathbf{O}_{i}\right\}$ on the upper surface of the lower disk is represented by ${ }^{i} \mathbf{p}_{p 1}$, while the position vector of any point $p_{2}$ in $\left\{\mathbf{O}_{1 i}\right\}$ on the lower surface of the upper disk is represented by ${ }^{1 i} \mathbf{p}_{p 2}$. According to the coordinate transformation, the position vector of any point $p_{2}$ in $\left\{\mathbf{O}_{i}\right\}$ on the lower surface of the upper disk is represented by ${ }^{i} \mathbf{p}_{p 2}$. The transformation can be described as

$$
{ }^{i} \mathbf{p}_{p 2}={ }_{1 i}^{i} \mathbf{R}^{1 i} \mathbf{p}_{p 2}+{ }^{i} \mathbf{p}_{1 i}
$$


where ${ }_{1 i}^{i} \mathbf{R}$ is the rotation matrix from $\left\{\mathbf{O}_{i}\right\}$ to $\left\{\mathbf{O}_{1 i}\right\}$ and ${ }^{i} \mathbf{p}_{1 i}$ is the position vector of $\left\{\mathbf{O}_{1 i}\right\}$ relative to $\left\{\mathbf{O}_{i}\right\}$. Therefore, the relationship between cable length and angles $\theta$ and $\varphi$ can be expressed as

$$
l=\left|{ }^{i} \mathbf{p}_{p 2}-{ }^{i} \mathbf{p}_{p 1}\right|,
$$

where $\mathbf{p}_{p 1}$ and $\mathbf{p}_{p 2}$, respectively, represent the intersection points between the cables, the upper surface of the lower disk, and the lower surface of the upper disk.

Taking the cable length in a single joint of the proximal segment as an example, the coordinates of the points $A_{1}, B_{1}$, $C_{1}$, and $D_{1}$ in $\left\{\mathbf{O}_{i}\right\}$ are represented by ${ }^{i} \mathbf{p}_{A 1}=(r, 0,0)$, ${ }^{i} \mathbf{p}_{B 1}=(0,-r, 0), \quad{ }^{i} \mathbf{p}_{C 1}=(-r, 0,0)$, and ${ }^{i} \mathbf{p}_{D 1}=(0, r, 0)$, while the coordinates of the points $A_{2}, B_{2}, C_{2}$, and $D_{2}$ in $\left\{\mathbf{O}_{1 i}\right\}$ are represented by ${ }^{1 i} \mathbf{p}_{A 2}=(r, 0,0),{ }^{1 i} \mathbf{p}_{B 2}=(0,-r, 0)$, ${ }^{1 i} \mathbf{p}_{C 2}=(-r, 0,0)$, and ${ }^{1 i} \mathbf{p}_{D 2}=(0, r, 0)$. Therefore, the relationship between the cable length and angles $\theta$ and $\varphi$ can be expressed as

$$
\left\{\begin{array}{l}
l_{A}=\left|{ }^{i} \mathbf{p}_{A 2}-{ }^{i} \mathbf{p}_{A 1}\right|=\sqrt{2 r^{2}-2 r^{2} c \varphi+h^{2}-2 r h \cdot s \varphi} \\
l_{B}=\left|\mathbf{p}_{B 2}-{ }^{i} \mathbf{p}_{B 1}\right|=\sqrt{(-r \cdot c \theta-h \cdot s \theta+r)^{2}+(r \cdot s \theta+h \cdot c \theta)^{2}} \\
l_{C}=\left|\mathbf{p}_{C 2}-{ }^{i} \mathbf{p}_{C 1}\right|=\sqrt{2 r^{2}-2 r^{2} c \varphi+h^{2}+2 r h \cdot s \varphi} \\
l_{D}=\left|{ }^{i} \mathbf{p}_{D 2}-{ }^{i} \mathbf{p}_{D 1}\right|=\sqrt{(r \cdot c \theta-h \cdot s \theta-r)^{2}+(-r \cdot s \theta+h \cdot c \theta)^{2}}
\end{array},\right.
$$

where $l_{A}, l_{B}, l_{C}$, and $l_{D}$ represent the cable length in the joint of the proximal segment, $c \theta=\cos \theta, s \theta=\sin \theta, c \varphi=\cos \varphi$, and $s \varphi=\sin \varphi$.

When angles $\theta$ and $\varphi$ are equal to zero, each cable length in the joint is equal to $h$, and the proximal segment keeps a straight configuration. According to equation (4), the length of cable $A$ and cable $C$ only depends on the angle $\varphi$, while the length of cable $B$ and cable $D$ only depends on the angle $\theta$. Consequently, during the 2-DOF bending motion of the proximal segment, a pair of antagonistic cable inputs only controls the 1-DOF rotation output, and the rotation output of each DOF of the joint is only determined by a single pair of antagonistic cable inputs. The 2-DOF bending motion in the proximal segment is completely decoupled. Similarly, the 2-DOF decoupled effect of the distal segment is the same as the decoupled effect of the proximal segment.

According to the literature $[37,38]$, if the sum of the cables length changes in each one pair of antagonistic cables is positive, the cables will not become slack. When the proposed joint bends from a straight configuration to an arbitrary bending configuration around axes $\mathbf{w}_{1}$ and $\mathbf{w}_{2}$, the relationship between the sum of the cable length changes in each one pair of antagonistic cables and bending angles is shown in Figure 7. The sum of length changes in the antagonistic cables is positive regardless of the bending angles. This indicates that the cables will not become slack when the proximal segment and the distal segment achieve, respectively, 2-DOF bending motion.
3.2. Robot Kinematics. Based on the joint kinematics, the kinematics of the cable-driven hyper-redundant robot is established. The mapping between the actuator space, joint space, and task space is also obtained [39]. The base coordinate system $\left\{\mathbf{O}_{0}\right\}$ is established at the center of the lower surface of the base disk, as shown in Figure 8. Axis $\boldsymbol{x}_{0}$ is parallel to the axis $\mathbf{w}_{1}$, and axis $\mathbf{z}_{0}$ is perpendicular to the lower surface of the base disk. According to the joint kinematics, the establishment principle of the coordinate systems $\left\{\mathbf{O}_{1}\right\}-\left\{\mathbf{O}_{n}\right\}$ in the proximal segment is the same as the coordinate system $\left\{\mathbf{O}_{m 1}\right\}-\left\{\mathbf{O}_{m n}\right\}$ in the distal segment. Therefore, the mapping between actuator space and joint space is first established. We assume that each segment of the robot contains $n$ identical joints. In the straight configuration, each cable length in the proximal and distal segment can be obtained as

$$
\left\{\begin{array}{l}
L_{p}=n h+(n+1) t, \\
L_{d}=2 n h+2(n+1) t,
\end{array}\right.
$$

where $L_{p}$ represents the initial length of each cable driving the proximal segment and $L_{d}$ represents the initial length of each cable driving the distal segment.

According to equation (2) and equation (3), the relationship between each cable length and bending angles in the arbitrary configurations can be derived as

$$
\left\{\begin{array}{l}
L_{P}^{\prime}=(n+1) t+n\left|{ }^{i} \mathbf{p}_{p 2}-{ }^{i} \mathbf{p}_{p 1}\right|, \\
L_{d}^{\prime}=L_{P}^{\prime}+(n+1) t+n\left|{ }^{j} \mathbf{p}_{p 4}-{ }^{j} \mathbf{p}_{p 3}\right|,
\end{array}\right.
$$

where $L_{p}^{\prime}$ represents the cables' length in the proximal segment and $L_{d}^{\prime}$ represents the cables' length in the distal segment. In the proximal segment, ${ }^{i} \mathbf{p}_{p 1}$ and ${ }^{i} \mathbf{p}_{p 2}$ are the intersection description between the cable on any side and the upper surface of the lower disk and the lower surface of the upper disk in the coordinate system $\left\{\mathbf{O}_{i}\right\}$, respectively. In the distal segment, and ${ }^{j} \mathbf{p}_{p 3}$ and ${ }^{j} \mathbf{p}_{p 4}$ are the intersection description between the cable on any side and the upper surface of the lower disk and the lower surface of the upper disk in the coordinate system $\left\{\mathbf{O}_{j}\right\}$, respectively. Therefore, the mapping between the actuator space and joint space can be established by equation (6). According to the transformation shown in Figure 8, the mapping between joint space and task space can be established. Hence, the homogeneous transformation matrix from the coordinate system $\left\{\mathbf{O}_{0}\right\}$ to the coordinate system $\left\{\mathbf{O}_{m n}\right\}$ can be written as

$$
{ }_{m n}^{0} \mathbf{T}={ }_{1}^{0} \mathbf{T} \times\left({ }_{i+1}^{i} \mathbf{T}\right)^{n} \times{ }_{m 1}^{n} \mathbf{T} \times\left({ }_{j+1}^{j} \mathbf{T}\right)^{n} .
$$

In the proposed cable-driven hyper-redundant robot, the adjacent two axes in each joint are perpendicular to different bending planes, which causes that the inverse kinematics is difficult to solve through the analytical method [40-42]. The Newton-Raphson iterative method can be used to solve the inverse kinematics, but it is not the research focus in this paper. 


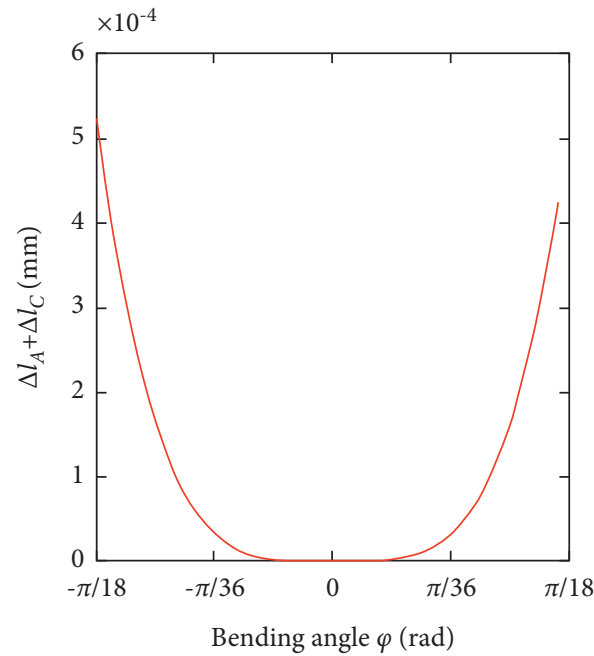

(a)

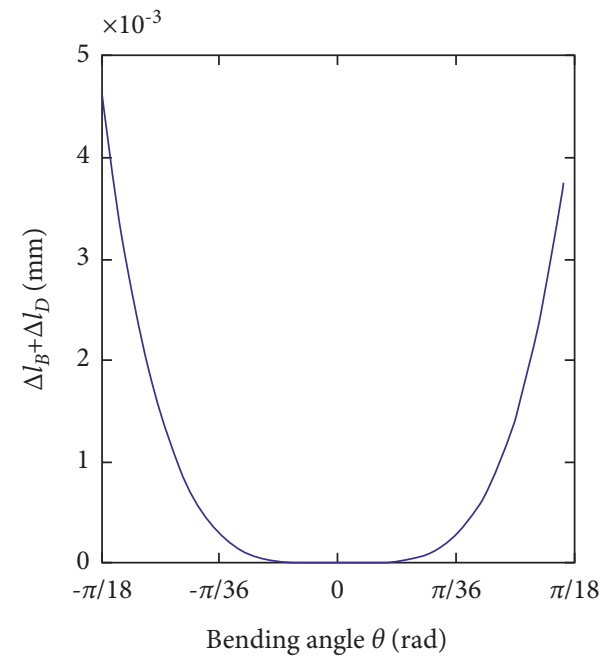

(b)

Figure 7: The sum of the antagonistic cables' length changes in any pair of cables. (a) Bending angle $\varphi$ (rad). (b) Bending angle $\theta$ (rad).

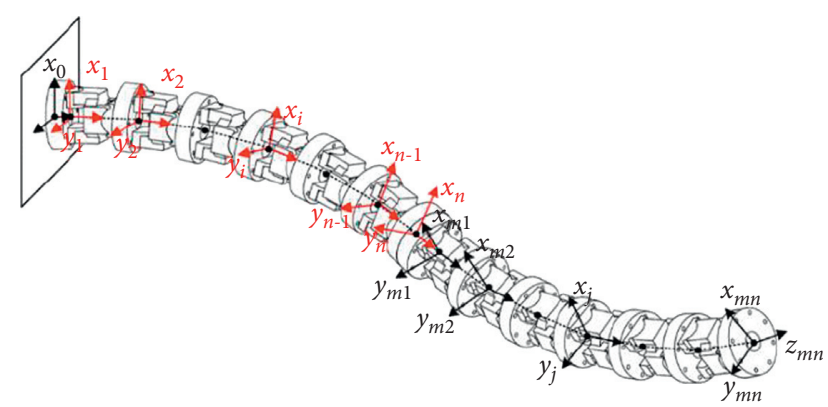

Figure 8: Coordinate system of the cable-driven hyper-redundant robot.

3.3. Workspace Analysis. The workspace of the cable-driven hyper-redundant robot is determined by the joint geometry, bending angles, and the joint number [43]. Based on the robot kinematics, the robot workspace is obtained. Figure 9(a) shows the workspace of the proximal segment, while Figure 9(b) shows nine bending configurations of the proximal segment when the joint angles $(\theta, \varphi)$ are, respectively, $(0,0),(0, \pi / 36),(0,-\pi / 36),(\pi / 36,0),(-\pi / 36,0)$, $(\pi / 36,-\pi / 36),(-\pi / 36, \pi / 36),(-\pi / 36,-\pi / 36)$, and $(\pi / 36$, $\pi / 36)$. Based on this, Figure $9(\mathrm{c})$ shows the workspace of the robot, while Figure 9(d) shows multiple bending configurations of the robot when joint angles $(\theta, \varphi, \alpha, \beta)$ are, respectively, $(0,0,0,0),(0,0,0, \pi / 36),(0,0,0,-\pi / 36),(0,0$, $\pi / 36,0),(0,0,-\pi / 36,0),(\pi / 36,0,0,0),(-\pi / 36,0,0,0),(0$ $-\pi / 36,0,0)$, and $(0, \pi / 36,0,0)$. According to the above analysis, the more the segment number is, the larger the workspace of the robot becomes.

\section{Kinematics Linear Fitting}

Based on equation (4) and equation (6), the relationship between each cable length and bending angles is the nonlinear function. However, the following kinematics linear fitting and error analysis will show that the relationship can be well fitted to a linear function in a certain range of joint variables, and the tightened amount of the cable on one side is almost equal to the released amount of the antagonistic cable when the robot configuration changes. Hence, any pair of antagonistic cables in the robot can be driven by a motor and a circle cable pulley, as shown in Figure 3(c), which not only simplifies the driving mechanism but also reduces the control complexity. The following contents are the kinematic linear fitting and error analysis in two segments. Based on the results, the driving mechanisms of the two segments are designed.

4.1. Kinematics Linear Fitting in the Proximal Segment. Since the 2-DOF bending motion in the proximal segment is decoupled, cable $A$ and cable $C$ are considered as an example to perform the kinematic linear fitting using the polynomial fitting method. The error values between the original and the fitting function are analyzed by the percentage error model. The percentage error $e_{l} \%(\varphi)$ is defined as

$$
e_{l} \%(\varphi)=100 \cdot\left|\frac{l(\varphi)-l^{\prime}(\varphi)}{l(\varphi)}\right|,
$$

where $l(\varphi)$ represents the original function and $l(\varphi)$ represents the fitting function. The fitting curve and error values between the original function and the fitting function of cable $A$ and cable $C$ are solved by the MATLAB curve fitting tool, as shown in Figure 10. The fitting functions of cable $A$ and cable $C$ are represented by $l_{A}^{\prime}(\varphi)=-4.237 \varphi+8$ and $l_{C}^{\prime}(\varphi)=4.237 \varphi+8$, respectively, as shown in Figure 10(a).

Within the range of bending angles shown in Table 1 , the maximum fitting error between the original function and fitting function is $0.025 \%$, and the maximum angle error of 


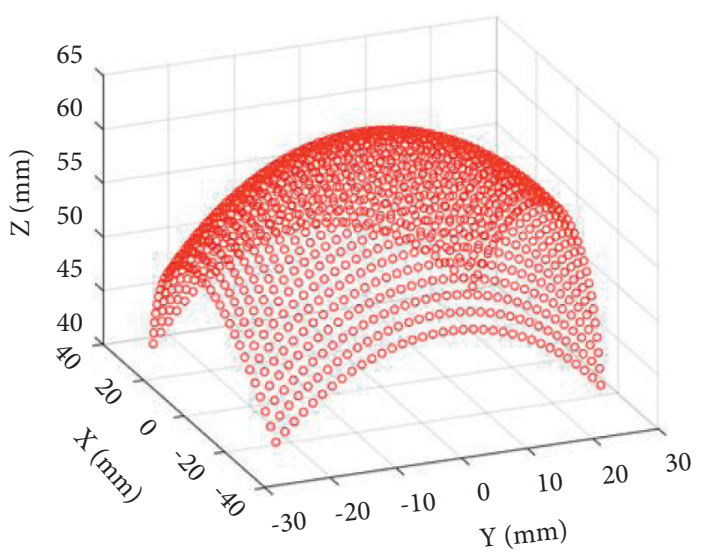

(a)

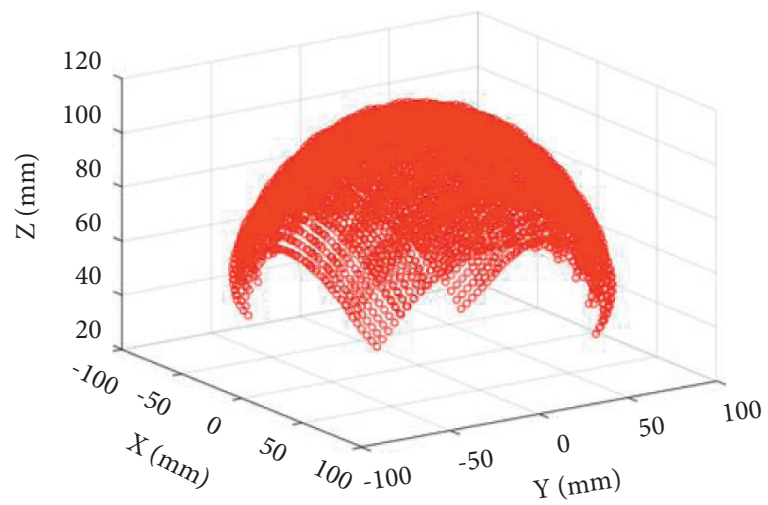

(c)

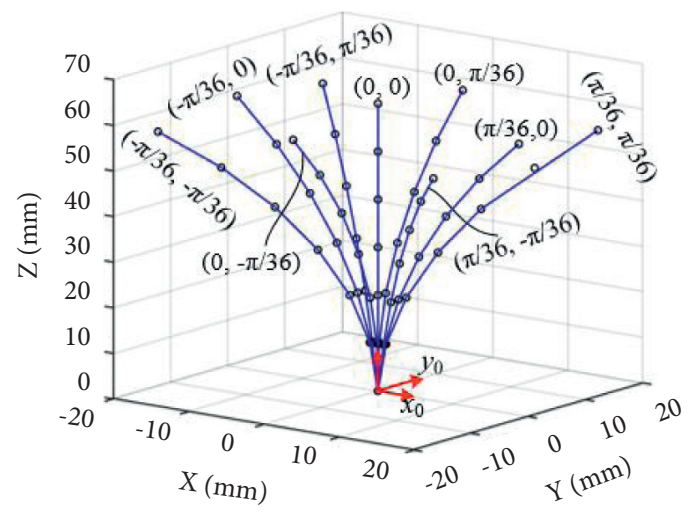

(b)

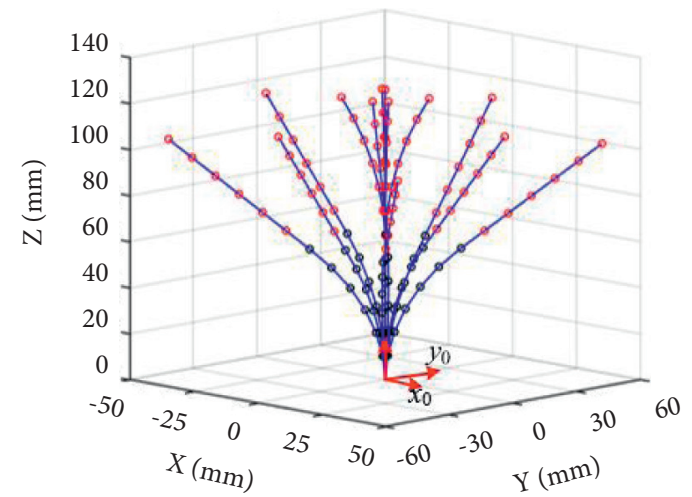

(d)

FIGURE 9: The workspace and bending configurations of the robot. (a) The workspace of the proximal section. (b) Nine bending configurations of the proximal section. (c) The robotic workspace. (d) Multiple bending configurations of the robot.

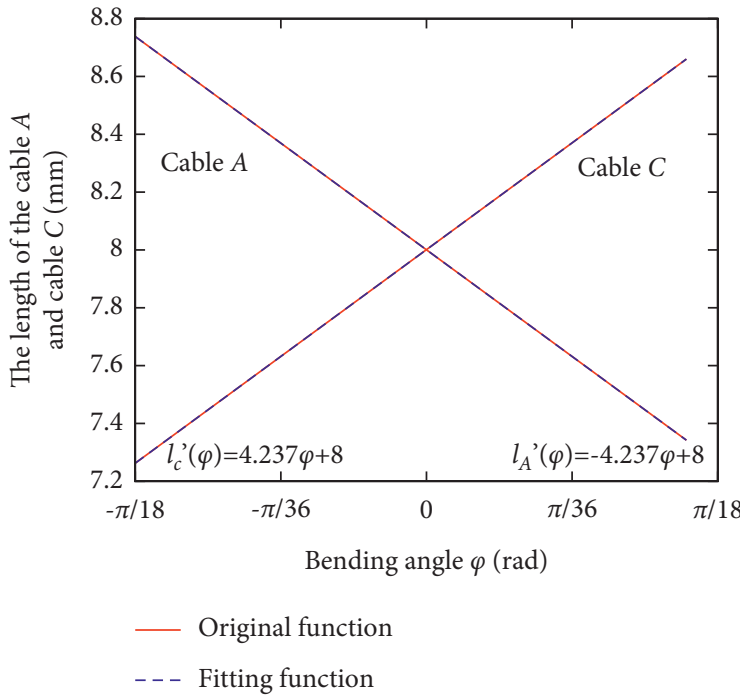

(a)

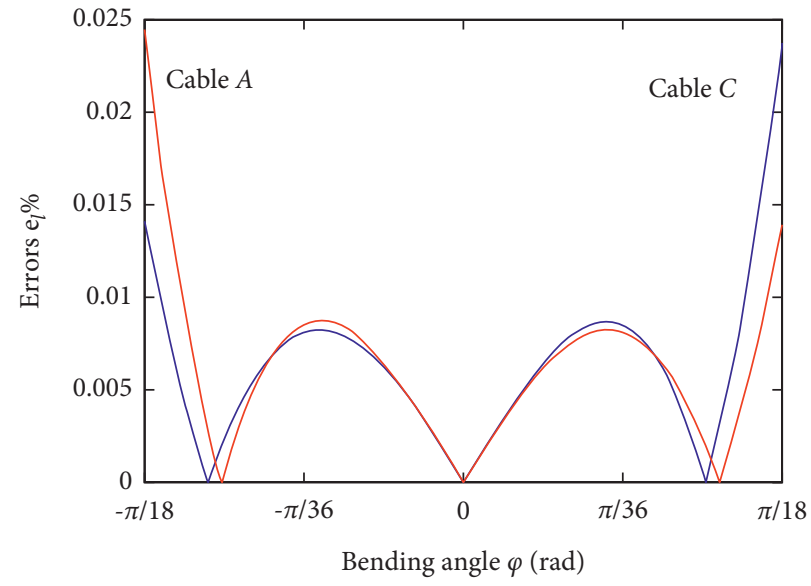

(b)

Figure 10: Cable A and cable C error between the original and fitted function. (a) Bending angle $\varphi$ (rad). (b) Bending angle $\theta$ (rad).

the end disk in the proximal segment is less than $0.15 \%$, as shown in Figure 10(b). Based on the same kinematics fitting method, the fitting functions of cable $B$ and cable $D$ are represented by $l_{B}^{\prime}(\theta)=-4.237 \theta+8$ and $l_{D}^{\prime}(\theta)=4.237 \theta+8$, respectively. Therefore, the relationship between each cable length and bending angles is linear through the special 
distribution of two rotation axes. It means the mapping between actuator space and joint space is simplified. Moreover, the 2-DOF bending motion of the proximal segment can be driven by two motors and two circular pulleys. Based on the literature [29], the robot motion accuracy is satisfied.

4.2. Kinematics Linear Fitting in the Distal Segment. According to equation (2) and equation (3), when the proximal segment undergoes 2 -DOF bending motion, the length of all eight cables will change. This means that the bending motion between the proximal segment and distal segment is coupled. Therefore, the kinematics linear fitting of cable $E$, cable $G$, cable $F$, and cable $H$ in proximal and distal segments should be considered to design the driving mechanism of the distal segment.

When the proximal segment keeps the straight configuration, and the distal segment keeps an arbitrary bending configuration, the relationship between the length of cable $E$, cable $G$, cable $F$, and cable $H$ and bending angles $\alpha$ and $\beta$ can be expressed as

$$
\left\{\begin{array}{l}
l_{E 1}=\sqrt{2 r^{2}-2 r^{2} c \alpha+h^{2}-2 r h \cdot s \alpha}, \\
l_{F 1}=\sqrt{(-r \cdot c \beta-h \cdot s \beta+r)^{2}+(r \cdot s \beta+h \cdot c \beta)^{2}}, \\
l_{G 1}=\sqrt{2 r^{2}-2 r^{2} c \alpha+h^{2}+2 r h \cdot s \alpha}, \\
l_{H 1}=\sqrt{(r \cdot c \beta-h \cdot s \beta-r)^{2}+(-r \cdot s \beta+h \cdot c \beta)^{2}}
\end{array},\right.
$$

where $l_{E 1}, l_{F 1}, l_{G 1}$, and $l_{H 1}$ represent the length of a single joint in the distal segment, $\mathrm{c} \alpha=\cos \alpha, \mathrm{s} \alpha=\sin \alpha, \mathrm{c} \beta=\cos \beta$, and $s \beta=\sin \beta$. According to equation (9), the 2 -DOF bending motions of the distal segment are decoupled when the proximal segment does not achieve the 2-DOF bending motion. In addition, the relationship between the cable length of the distal segment and the bending angles $\alpha$ and $\beta$ is also approximately linear.

If the proximal segment achieves 2-DOF bending motion, the lengths of cable $E$, cable $G$, cable $F$, and cable $H$ will also change. Hence, the cable length change relationship in the proximal segment should be considered to achieve the kinematics linear fitting of the distal segment. According to equation (2) and equation (3), the relationship between the length of cable $E$, cable $G$, cable $F$, and cable $H$ and bending angles $\theta$ and $\varphi$ can be calculated as

$$
\left\{\begin{array}{l}
l_{E 2}=\left|{ }^{i} \mathbf{p}_{E 2}-{ }^{i} \mathbf{p}_{E 1}\right|, \\
l_{F 2}=\left|{ }^{i} \mathbf{p}_{F 2}-{ }^{i} \mathbf{p}_{F 1}\right|, \\
l_{G 2}=\left|{ }^{i} \mathbf{p}_{G 2}-{ }^{i} \mathbf{p}_{G 1}\right|, \\
l_{H 2}=\left|{ }^{i} \mathbf{p}_{H 2}-{ }^{i} \mathbf{p}_{H 1}\right|,
\end{array}\right.
$$

where $l_{E 2}, l_{F 2}, l_{G 2}$, and $l_{H 2}$ represent the cable length of the single joint in the proximal segment. Since the 2-DOF bending motion in the proximal segment has similar kinematics, cable $E$ and cable $G$ are considered as an example to perform the kinematic linear fitting using the polynomial fitting method. Within the range of bending angles shown in Table 1, the kinematics linear fitting results of cable $E$ and cable $G$ in each joint of the proximal segment are shown in Figure 11.

Similarly, the MATLAB curve fitting tool is also used to solve the fitting function. The fitting functions of cable $E$ and cable $G$ are represented by $l_{E}^{\prime}(\theta, \varphi)=-2.996 \theta-2.996 \varphi+8$, $l_{G}^{\prime}(\theta, \varphi)=2.996 \theta+2.996 \varphi+8$. The percentage error $e_{l} \%$ is redefined as

$$
e_{l} \%(\theta, \varphi)=100 \cdot\left|\frac{l(\theta, \varphi)-l^{\prime}(\theta, \varphi)}{l(\theta, \varphi)}\right|,
$$

where $l(\theta, \varphi)$ represents the original function and $l(\theta, \varphi)$ represents the fitting function.

According to equation (11), the maximum fitting errors between the original function and fitting function of cable $E$ and cable $G$ are less than $0.04 \%$, while the maximum fitting errors of the cable $F$ and cable $H$ are less than $0.25 \%$, as shown in Figure 12. Therefore, the relationship between the length of cable $E$, cable $G$, cable $F$, and cable $H$, and bending angles can be approximately linear. This indicates that the mapping between actuator space and joint space in the distal segment is also simplified. Therefore, the 2-DOF bending motion of the distal segment can be achieved by two motors and two circle pulleys. Based on the literature [29], the robot motion accuracy is satisfied.

\section{Experiment Validation}

In this section, a 4-DOF cable-driven hyper-redundant robot prototype is established to validate the robot design. The proposed robot includes the proximal segment, the distal segment, and the driving mechanism, as shown in Figure 13(a). The total length of the proximal segment and the distal segment is $131 \mathrm{~mm}$. The cables' diameter is $0.4 \mathrm{~mm}$. The driving mechanism of the robot prototype includes a guiding device, a motor driving device, and a cable tension adjusting device, as shown in Figure 13(b). The rated speed of the motor is $10 \mathrm{r} / \mathrm{min}$, and the rated torque is $70 \mathrm{~kg} \mathrm{~cm}$. The range of the force sensor is $0-10 \mathrm{~kg}$ with an accuracy of $0.03 \%$.

According to the kinematic linear fitting relationship in Section 4, the driving mechanisms of the proximal and distal segments are the same. For the proximal segment, cable $A$ and cable $C$ are the two ends of one cable that is driven by motor 1 to control the proximal segment bending in the $x_{0} z_{0}$ plane. Cable $B$ and cable $D$ are also the two ends of one cable that is driven by motor 2 to control the proximal segment bending in the $y_{0} z_{0}$ plane.

For the distal segment, cable $E$ and cable $G$ are the two ends of one cable that is driven by motor 3 to control the distal segment bending in the $x_{m 1} z_{m 1}$ plane. Cable $F$ and cable $H$ are the two ends of one cable that is driven by motor 4 to control the distal segment bending in the $y_{m 1} z_{m 1}$ plane. The two ends of each cable are fixedly connected to the end disks of the proximal segment and distal segment through knotting. The middle of each cable passes through each joint disk and winds around the guide device, driving device, and tension adjusting device, as shown in Figure 13(b). Each cable tension is 


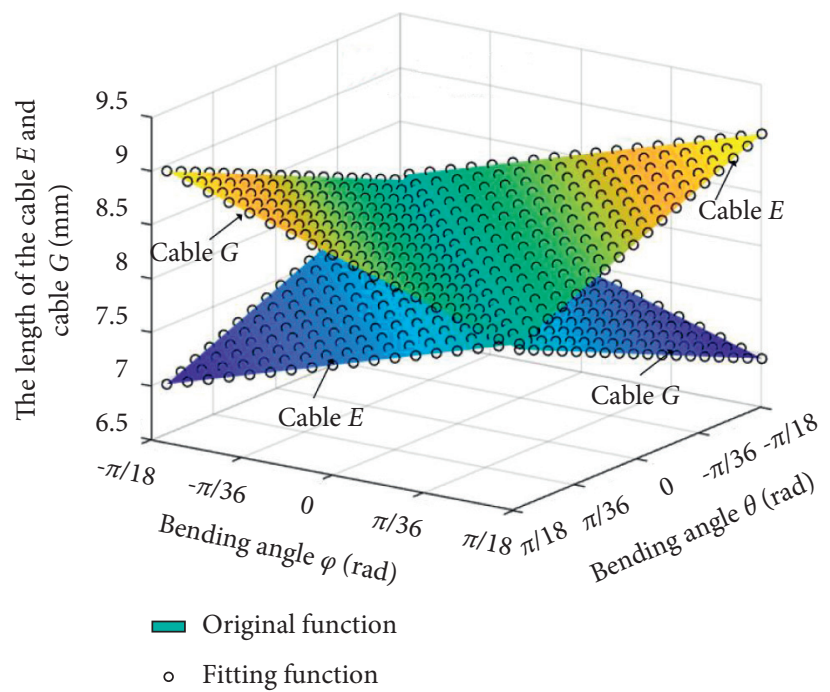

FIgURE 11: Kinematics linear fitting analysis of cable E and cable G.

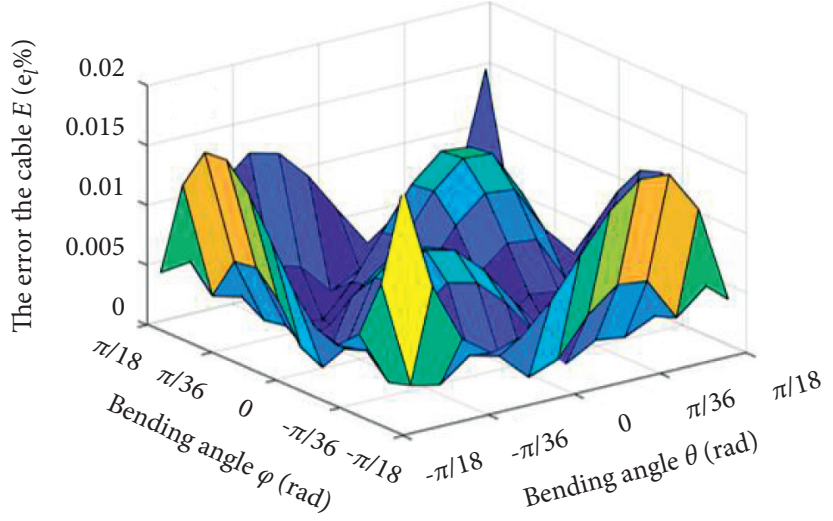

(a)

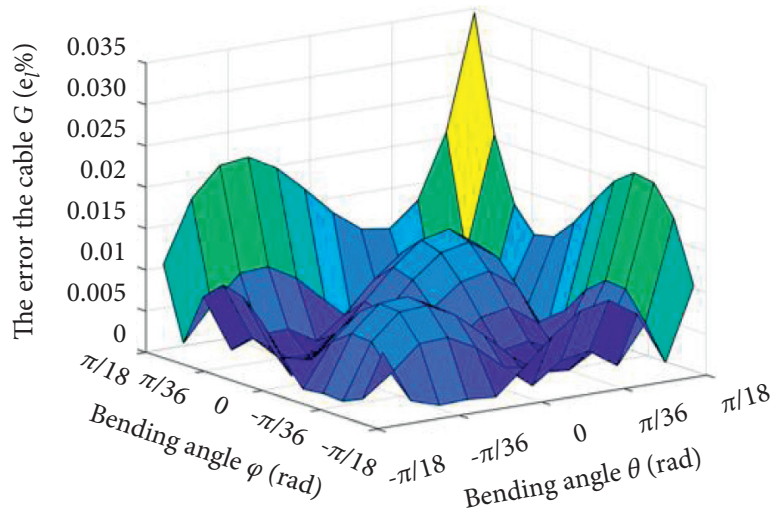

(c)

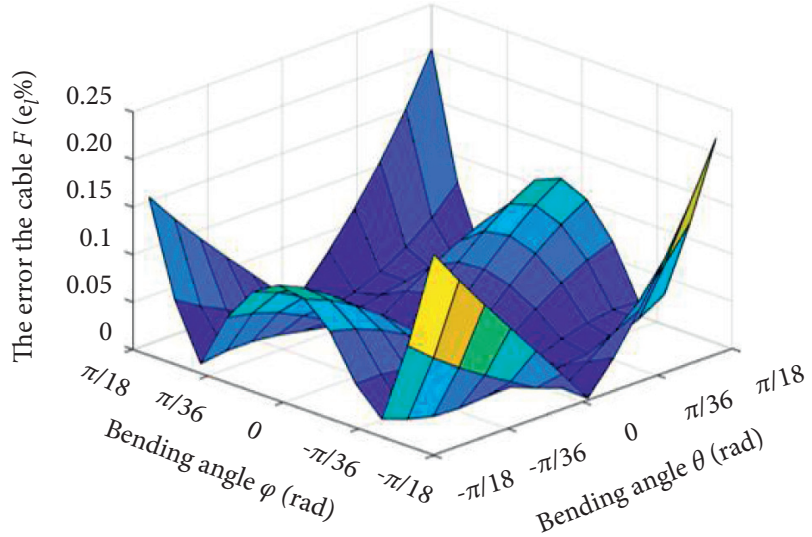

(b)

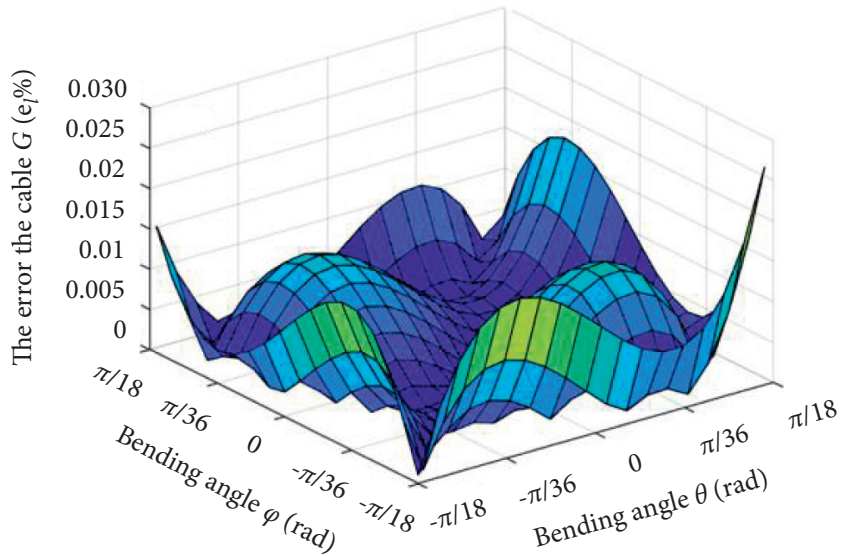

(d)

FIgURE 12: Kinematics linear fitting error analysis of cables E, G, F, and $\mathrm{H}$.

adjusted by changing the position of the sliding block. The cable tension values are tested by the tension sensors. The following experiments include the free bending motion test, the cable tension test, and payload experiments.
5.1. Free Bending Motion. In this section, the multi-DOF bending motions of the proximal segment and distal segment have experimented, as shown in Figure 14. 


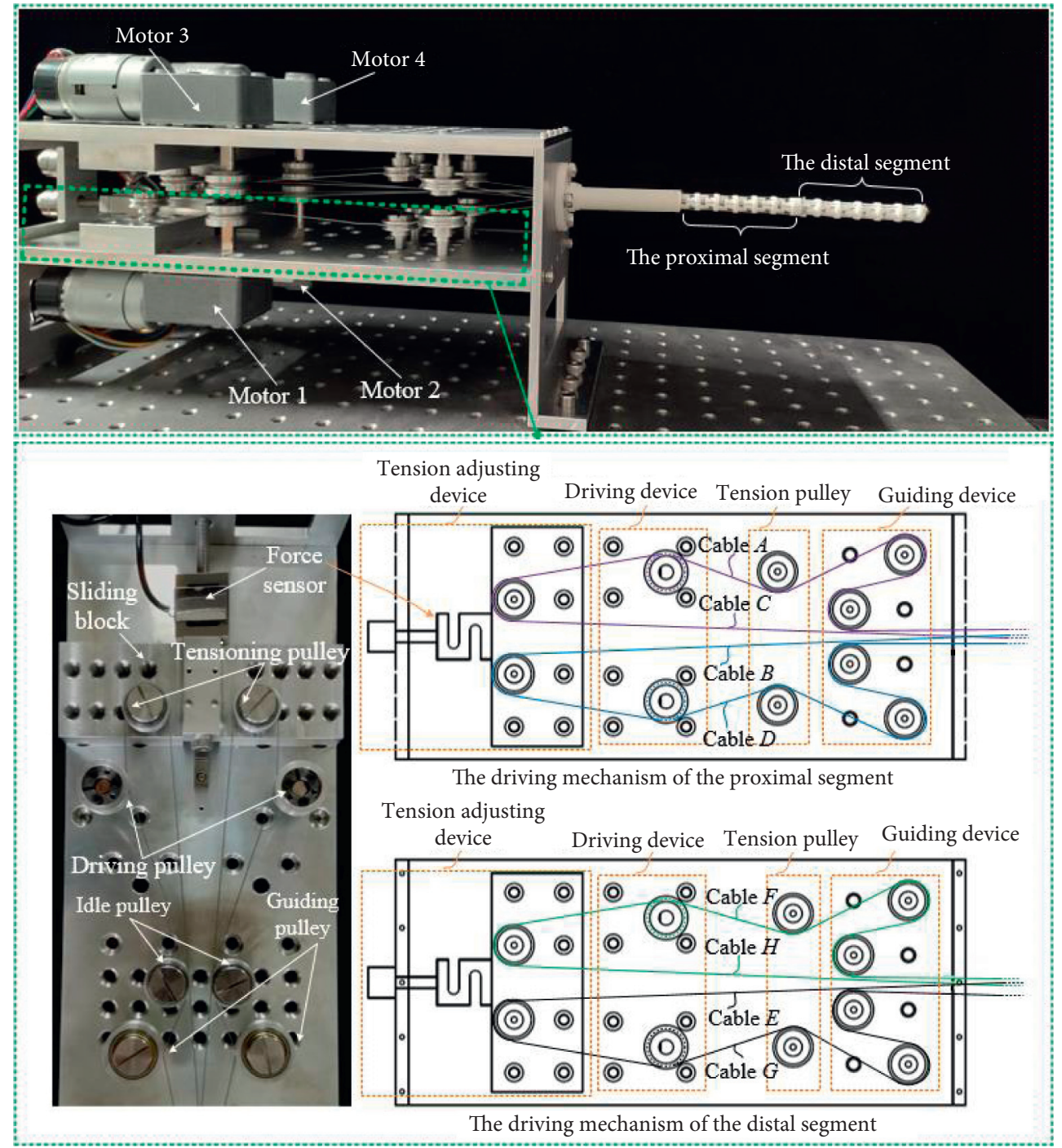

Figure 13: Cable-driven hyper-redundant robot prototype.

When the distal segment keeps a straight configuration, the bending motion of the proximal segment in the $x_{0} z_{0}$ plane requires the coordinated work of motor 1 , motor 3 , and motor 4 . Motor 2 does not work to ensure that the cable lengths of cable $B$ and cable $D$ are unchanged. The bending configuration outputs of the proximal segment in the $x_{0} z_{0}$ plane are only determined by the inputs of motor 1 . Motor 3 and motor 4 are driven to keep the straight configuration of the distal segment. The bending configurations are shown in Figures 14(a)-14(c).

When the distal segment keeps a straight configuration, the bending motion of the proximal segment in the $y_{0} z_{0}$ plane requires the coordinated work of motor 2, motor 3, and motor 4 . Motor 1 does not work to ensure that the lengths of cable $A$ and cable $B$ are unchanged. The bending configuration outputs of the proximal segment in the $x_{0} z_{0}$ plane are only determined by the inputs of motor 2. Motor 3 and motor 4 are driven to keep the straight configuration of the distal segment. The bending configurations are shown in Figures $14(\mathrm{~d})-14(\mathrm{~g})$.
When the proximal segment keeps a straight configuration, the bending motion of the distal segment in the $x_{m 1} z_{m 1}$ plane only requires motor 3 working to change the length of cable $E$ and cable $G$. The other motors do not work to ensure that the cable lengths of cable $A$, cable $C$, cable $E$, cable $G$, cable $F$, and cable $H$ are unchanged. The bending configuration outputs of the proximal segment in the $x_{m 1} z_{m 1}$ plane are only determined by the inputs of motor 3. Motor 1, motor 2 , and motor 4 are not driven to keep the straight configuration of the proximal segment. The bending configurations are shown in Figures 14(h)14(j).

Similarly, the bending motion of the distal segment in the $y_{m 1} z_{m 1}$ plane only requires motor 4 working to change the length of cable $F$ and cable $H$. Besides, to verify the multi-DOF bending motion of the robot, we consider the bending configuration of the proximal segment in the $x_{0} z_{0}$ plane and the bending configuration of the distal segment in the $x_{m 1} z_{m 1}$ plane as an example, as shown in Figures $14(\mathrm{k})-14(\mathrm{n})$. 


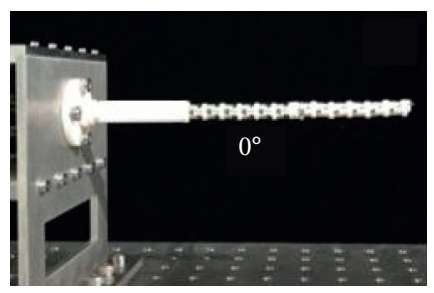

(a)

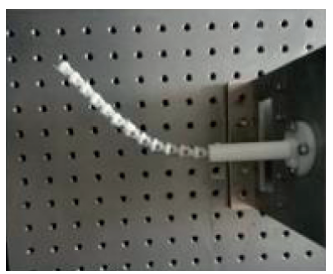

(e)

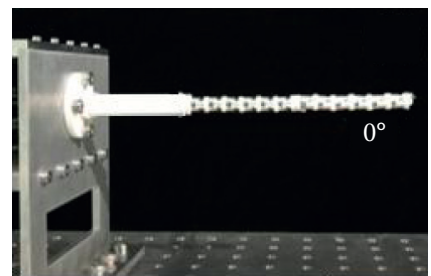

(h)

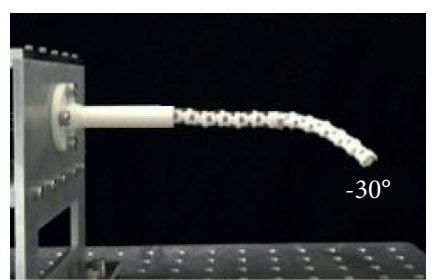

(i)

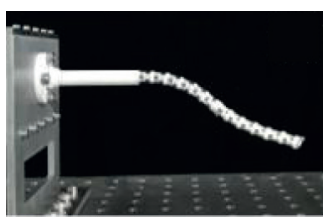

(l)

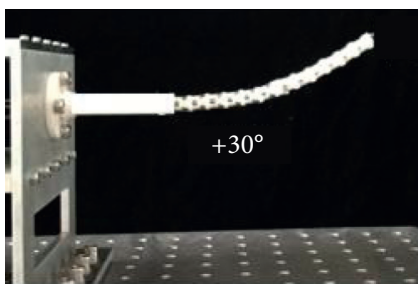

(b)

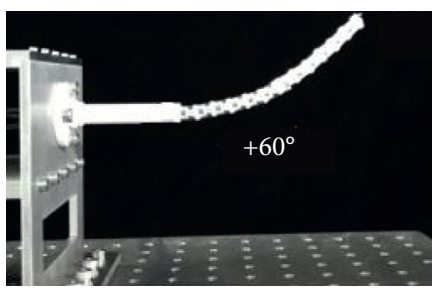

(c)

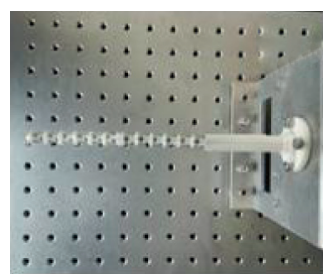

(d)

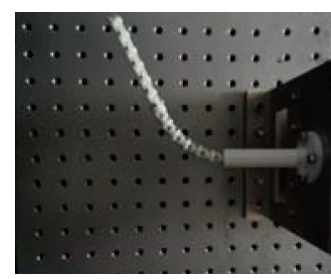

(f)

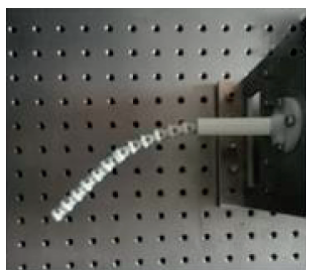

(g)

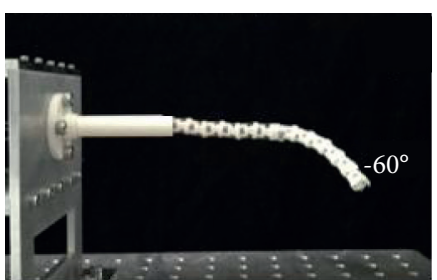

(j)

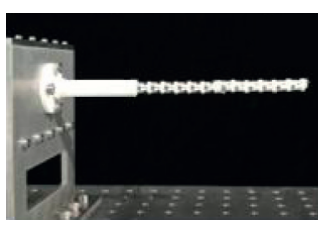

(k)

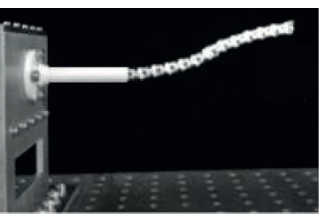

(n)

FIgure 14: Robot bending motion experiments.

5.2. Cable Tension Test. During the multiple bending motions, the cable average tension curves are used to illustrate the design rationalization of the driving mechanism for the proximal segment and distal segment [44]. For the proximal segment, the bending configuration in the $x_{0} z_{0}$ plane is determined by the angle $\theta$, while the bending configuration in the $y_{0} z_{0}$ plane is determined by the angle $\varphi$. When the distal segment keeps a straight configuration and the proximal segment keeps a bending configuration in the $x_{0} z_{0}$ plane and $y_{0} z_{0}$ plane, the average cable tension of each cable varies with the bending angles $\theta$ and $\varphi$, as shown in Figure 15.

Within the joint angle ranges of $[-\pi / 18, \pi / 18]$, when only the proximal segment bends in the $x_{0} z_{0}$ plane, the average tension of cable $A$ and cable $C$ in the proximal segment varies in the range of $13 \mathrm{~N}-15 \mathrm{~N}$, as shown in Figure 15(a). When only the proximal segment bends in the $y_{0} z_{0}$ plane, the average tension of cable $B$ and cable $D$ in the proximal segment changes within the range of $10 \mathrm{~N}-13 \mathrm{~N}$, as shown in Figure 15(b). For the cables of the distal segment, the average tension of the cable $E$, cable $G$, cable $F$, and cable $H$ varies in the range of $8 \mathrm{~N}-10 \mathrm{~N}$ and $6 \mathrm{~N}-10 \mathrm{~N}$. Since the 2DOF bending motion of the proximal segment will change the length of each cable driving the distal segment, the average tension of each cable driving the distal segment will increase.

When the proximal segment keeps a straight configuration and the distal segment keeps a bending configuration in the $x_{m 1} z_{m 1}$ plane and $y_{m 1} z_{m 1}$ plane, the average cable tension of each cable varies with the joint angles $\alpha$ and $\beta$, as shown in Figure 16. For the proximal segment, the bending configuration in the $x_{m 1} z_{m 1}$ plane is determined by the angle $\beta$, while the bending configuration in the $y_{m 1} z_{m 1}$ plane is determined by the angle $\alpha$.

Within the range of the bending angles of $[-\pi / 18, \pi / 18]$, when only the distal segment bends in the $x_{m 1} z_{m 1}$ plane, the average tension of the cable $F$ and cable $H$ in the distal segment varies in the range of $10 \mathrm{~N}-12 \mathrm{~N}$, as shown in Figure 16(a). When only the distal segment bends in the $y_{m 1} z_{m 1}$ plane, the average tension of cable $E$ and cable $G$ in the distal segment varies in the range of $11 \mathrm{~N}-13 \mathrm{~N}$, as shown in Figure 16(b). For the cables of the proximal segment, the average tension of cable $A$, cable $B$, cable $C$, and cable $D$ varies in the range of $12 \mathrm{~N}-15 \mathrm{~N}$. Therefore, the phenomenon of the cables slack does not appear during the multiDOF bending motion. The results indicate that the driving 


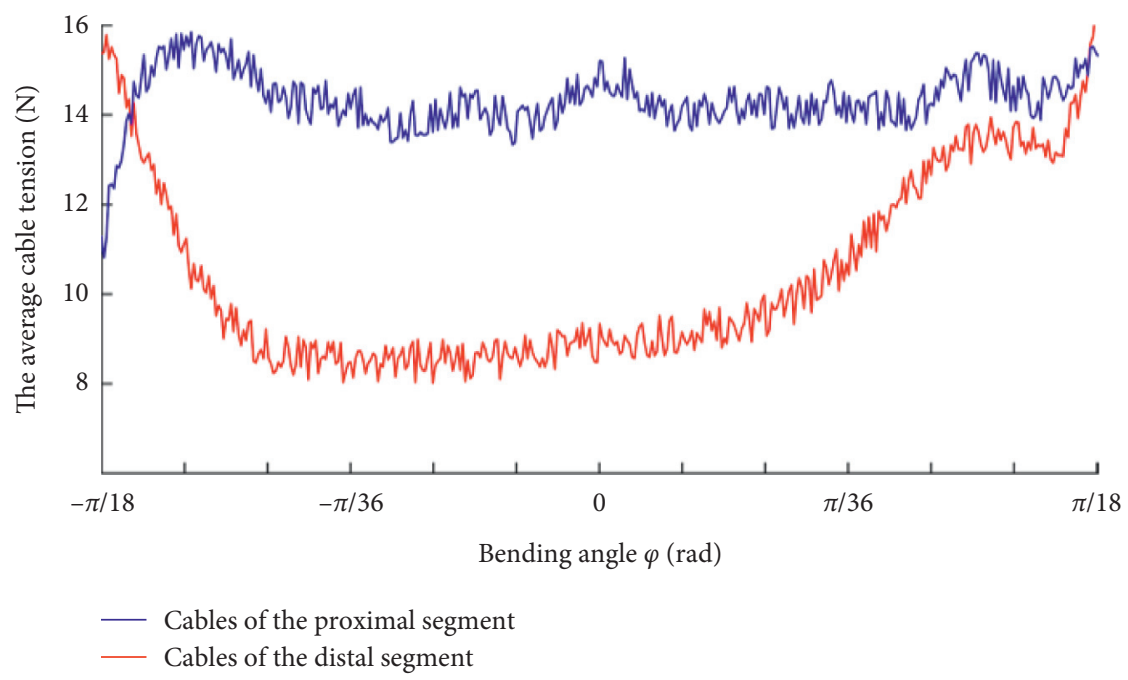

(a)

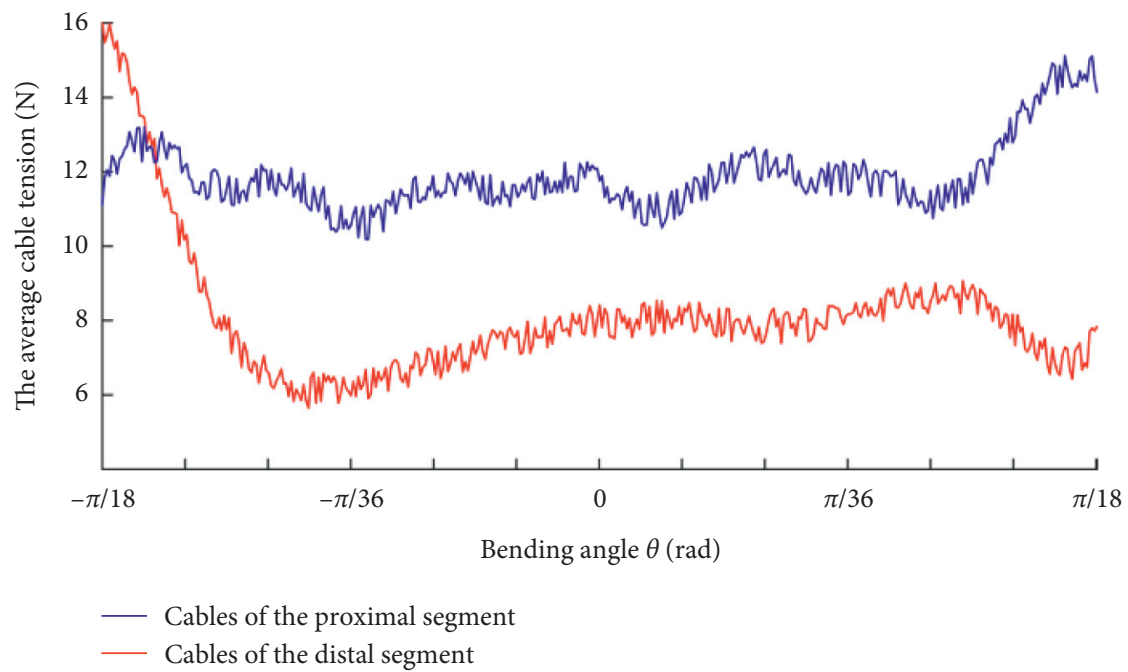

(b)

FIgURE 15: Cable tension during the bending motion of the proximal segment. (a) Bending angle $\varphi$ (rad). (b) Bending angle $\theta$ (rad).

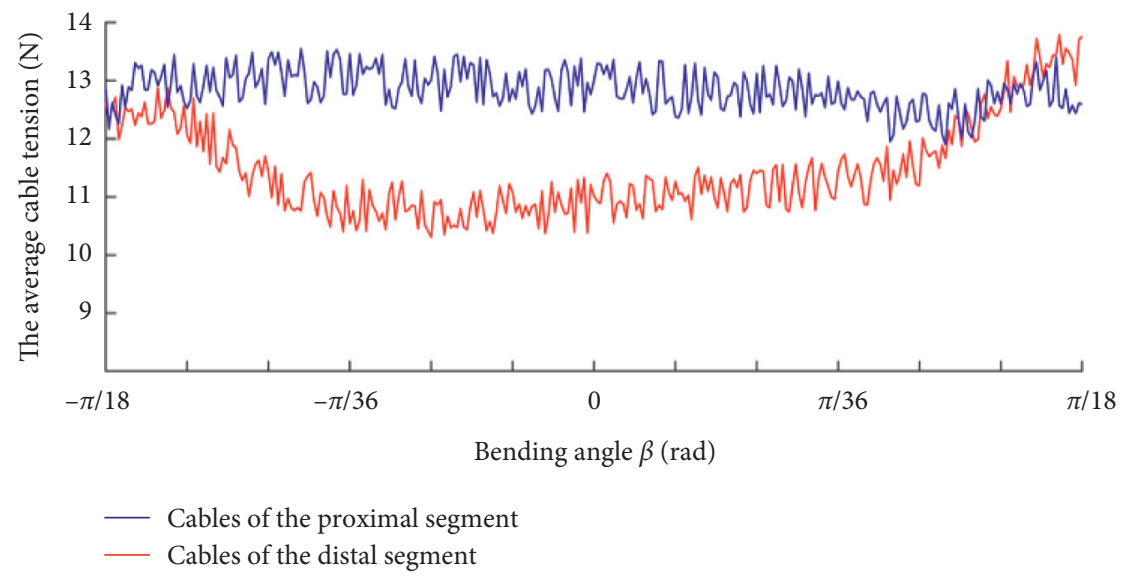

(a)

Figure 16: Continued. 


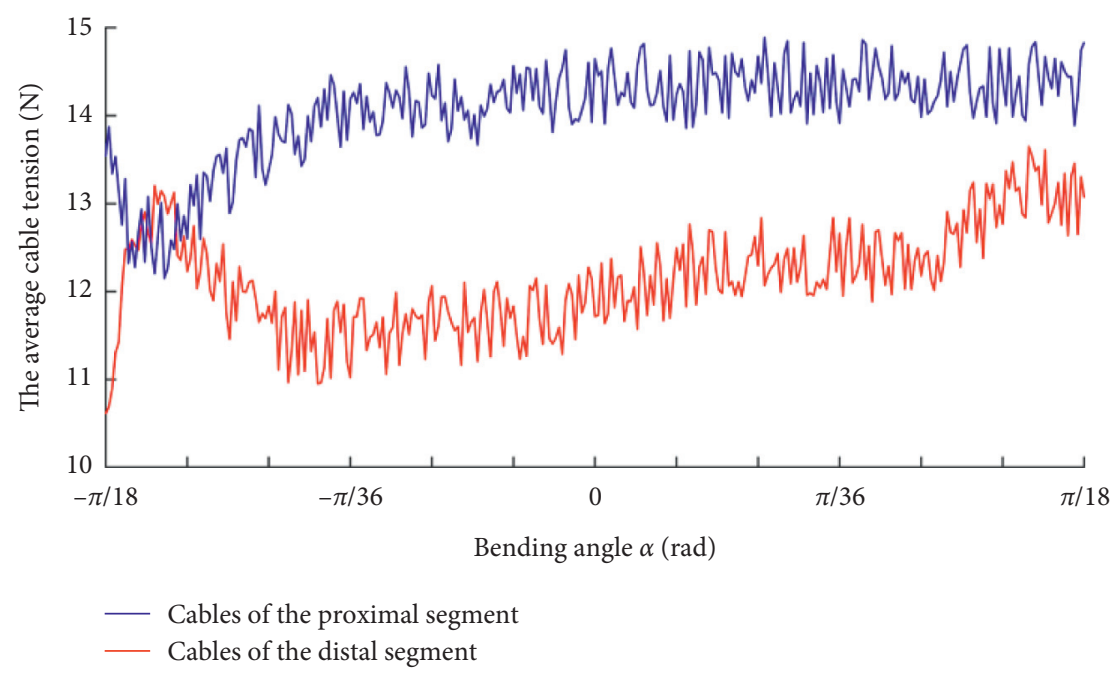

(b)

Figure 16: Cable tension during the bending motion of the distal segment. (a) Bending angle $\beta$ (rad). (b) Bending angle $\alpha$ (rad).

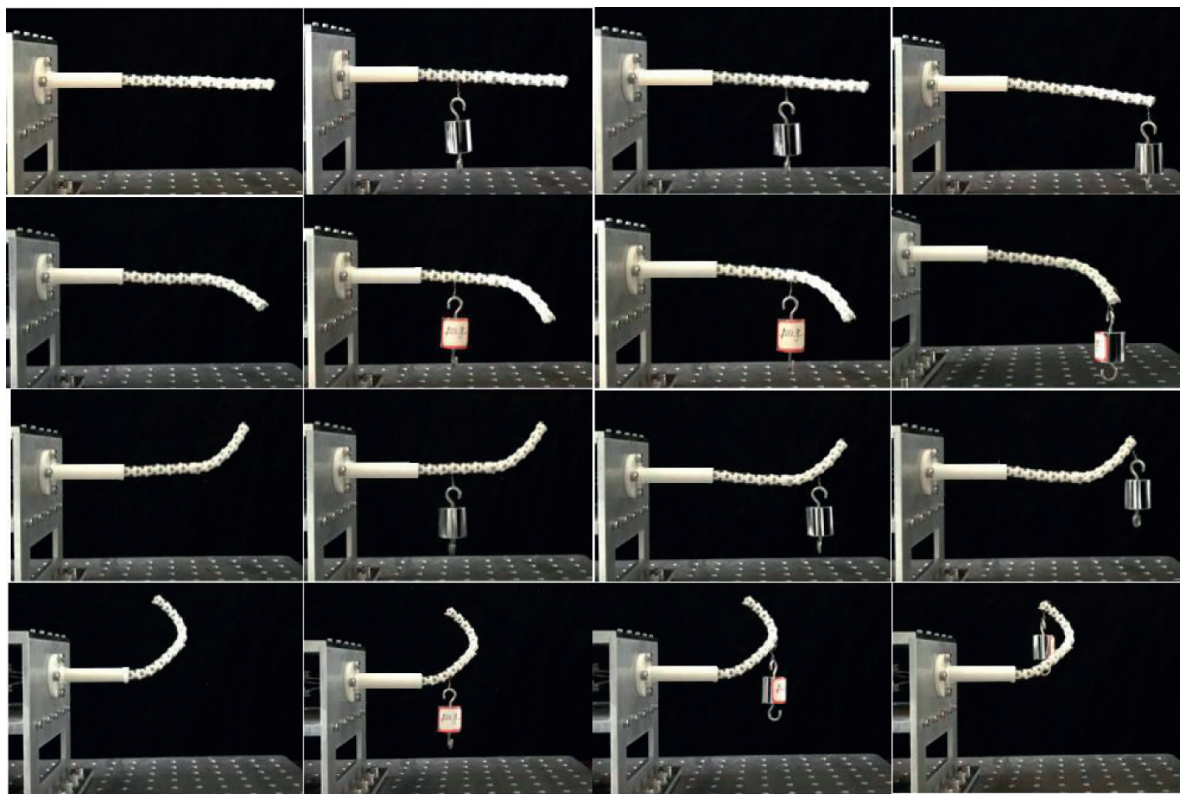

Figure 17: The $1 \mathrm{~N}$ payload experiments in the different positions of the robot.

mechanism design of the proximal and distal segments is reasonable.

5.3. Payload Experiments. When most of the prior cabledriven hyper-redundant robots are subjected to small external disturbance, the robots easily appear in the $S$ configuration and even other uneven configurations [45]. In this section, a $1 \mathrm{~N}$ weight is loaded at different positions of different bending configurations in the proposed robot, as shown in Figure 17. According to the observation, the proposed robot does not appear in the $S$ configuration, which means the proposed robot can resist small external disturbances. Since there are eight cables in the proximal segment and four cables in the distal segment, the load capacity of the proximal segment is stronger than the load capacity of the distal segment. Besides, when the load position keeps moving away from the driving mechanism, the deformation of the terminal position of the robot becomes larger. During the payload experiments, it can be easily known that the inevitable clearance between the cables and the cable holes, and the assembly errors of the initial configuration of each joint will aggravate the deformation of the robot under the external disturbance. In addition, the robot load capacity is also determined by the robot prototype structure parameters. 


\section{Conclusions and Future Works}

In this paper, a novel 2-DOF decoupled joint is first proposed. A 4-DOF cable-driven hyper-redundant robot prototype is developed based on the 2-DOF decoupled joint. The kinematics model is established and the workspace is analyzed. The kinematic fitting approach is presented for both proximal and distal segments. The mapping between actuator space and joint space is simplified through the kinematics linear fitting and error analysis. It means that the driving mechanism design of the robot is also simplified. Experiment results indicated that the 2-DOF bending motion of each segment is decoupled. According to the average cable tension and the payload experiments, the proposed robot design is feasible, and the hyper-redundant robot does not appear in the $S$ configuration when $1 \mathrm{~N}$ weight is loaded at different positions of different bending configurations in the proposed robot. In future research, the tip position and shape under the external forces and the position accuracy analysis of the robot will be analyzed and evaluated in detail. Besides, the method avoiding the kinematic coupling between the proximal segment and distal segment is also a research focus in the future.

\section{Data Availability}

The data used to support the findings of this study are included within the article.

\section{Conflicts of Interest}

The authors declare no conflicts of interest.

\section{Acknowledgments}

This work was supported by the National Natural Science Foundation of China (project nos. 51805047 and 52175003), Natural Science Foundation of Hunan Province (project no. 2021JJ40259), Outstanding Youth Program of Hunan Education Department (project nos. $20 \mathrm{~B} 307$ and 20B017), Open Research Project of the State Key Laboratory of Industrial Control Technology, and Zhejiang University, China (project no. ICT2021B02).

\section{References}

[1] O. M. Omisore, S. Han, J. Xiong, H. Li, Z. Li, and L. Wang, “A review on flexible robotic systems for minimally invasive surgery," IEEE Transactions on Systems, Man, and Cybernetics: Systems, pp. 1-14, 2020.

[2] C. Yang, S. Geng, I. Walker et al., "Geometric constraintbased modeling and analysis of a novel continuum robot with shape memory alloy initiated variable stiffness," The International Journal of Robotics Research, vol. 39, no. 14, pp. 1620-1634, 2020.

[3] H. Yuan, L. Zhou, and W. Xu, "A comprehensive static model of cable-driven multi-section continuum robots considering friction effect," Mechanism and Machine Theory, vol. 135, pp. 130-149, 2019.

[4] J. Wang, S. Wang, J. Li, X. Ren, and R. M. Briggs, "Development of a novel robotic platform with controllable stiffness manipulation arms for laparoendoscopic single-site surgery (LESS)," International Journal of Medical Robotics and Computer Assisted Surgery, vol. 14, no. 1, Article ID e1838, 2018.

[5] K. Xu, J. Zhao, and M. Fu, "Development of the SJTU unfoldable robotic system (SURS) for single port laparoscopy," IEEE, vol. 20, no. 5, pp. 2133-2145, 2014.

[6] L. Huang, L. Yin, B. Liu, and Y. Yang, "Design and error evaluation of planar 2DOF remote center of motion mechanisms with cable transmissions," Journal of Mechanical Design, vol. 143, no. 1, 2021.

[7] S. Kolachalama and S. Lakshmanan, "Continuum robots for manipulation applications: a survey," Journal of Robotics, vol. 2020, Article ID 4187048, 19 pages, 2020.

[8] M. H. Korayem, A. Zehfroosh, H. Tourajizadeh, and S. Manteghi, "Optimal motion planning of non-linear dynamic systems in the presence of obstacles and moving boundaries using SDRE: application on cable-suspended robot," Nonlinear Dynamics, vol. 76, no. 2, pp. 1423-1441, 2014.

[9] M. H. Korayem and M. Bamdad, "Dynamic load-carrying capacity of cable-suspended parallel manipulators," International Journal of Advanced Manufacturing Technology, vol. 44, no. 7-8, pp. 829-840, 2009.

[10] M. H. Korayem, M. Bamdad, H. Tourajizadeh, A. H. Korayem, and S. Bayat, "Analytical design of optimal trajectory with dynamic load-carrying capacity for cablesuspended manipulator," International Journal of Advanced Manufacturing Technology, vol. 60, no. 1, pp. 317-327, 2012.

[11] L. Huang, Y. Yang, J. Xiao, and P. Su, "Type synthesis of 1R1T remote center of motion mechanisms based on pantograph mechanisms," Journal of Mechanical Design Transactions of ASME, vol. 138, no. 1, Article ID 014501, 2016.

[12] F. Jelínek, E. A. Arkenbout, P. W. Henselmans, R. Pessers, and P. Breedveld, "Classification of joints used in steerable instruments for minimally invasive surgery-a review of the state of the art," Journal of Medical Devices, vol. 9, no. 1, 2015.

[13] M. C. Lei and R. Du, "Geometry modeling and simulation of the wire-driven bending section of a flexible ureteroscope," in Proceedings of the World Congress on Engineering and Computer Science Year, vol. 2, San Francisco, USA, October 2017.

[14] Z. Wang, T. Wang, B. Zhao et al., "Hybrid adaptive control strategy for continuum surgical robot under external load," IEEE Robotics and Automation Letters, vol. 6, no. 2, pp. 1407-1414, 2021.

[15] A. Gao, J. Li, Y. Zhou, Z. Wang, and H. Liu, "Modeling and task-oriented optimization of contact-aided continuum robots," IEEE, vol. 25, no. 3, pp. 1444-1455, 2020.

[16] W. S. Rone and P. Ben-Tzvi, "Mechanics modeling of multisegment rod-driven continuum robots," Journal of Mechanisms and Robotics, vol. 6, no. 4, 2014.

[17] Y. J. Kim, S. Cheng, S. Kim, and K. Iagnemma, "A stiffnessadjustable hyperredundant manipulator using a variable neutral-line mechanism for minimally invasive surgery," IEEE Transactions on Robotics, vol. 30, no. 2, pp. 382-395, 2013.

[18] J. Kim, S. I. Kwon, Y. Moon, and K. Kim, "Cable-movable rolling joint to expand workspace under high external load in a hyper-redundant manipulator," IEEE, 2021.

[19] W. Xu, T. Liu, and Y. Li, "Kinematics, dynamics, and control of a cable-driven hyper-redundant manipulator," IEEE, vol. 23, no. 4, pp. 1693-1704, 2018. 
[20] A. Kanada and T. Mashimo, "Switching between continuum and discrete states in a continuum robot with dislocatable joints," IEEE Access, vol. 9, pp. 34859-34867, 2021.

[21] W. Shen, G. Yang, T. Zheng, Y. Wang, K. Yang, and Z. Fang, "An accuracy enhancement method for a cable-driven continuum robot with a flexible backbone," IEEE Access, vol. 8, pp. 37474-37481, 2020.

[22] M. Dehghani and S. A. A. Moosavian, "Dynamics modeling of a continuum robotic arm with a contact point in planar grasp," Journal of Robotics, vol. 2014, Article ID 308283, 13 pages, 2014.

[23] Z. Li, M. Zin Oo, V. Nalam et al., "Design of a novel flexible endoscope-cardioscope," Journal of Mechanisms and Robotics, vol. 8, no. 5, 2016.

[24] X. Dong, D. Axinte, D. Palmer et al., "Development of a slender continum robotic system for on-wing inspection/repair of gas turbine engines," Robotics and Computer-Integrated Manufacturing, vol. 44, pp. 218-229, 2017.

[25] X. Jin, J. Zhao, M. Feng, L. Hao, and Q. Li, "Snake-like surgical forceps for robot-assisted minimally invasive surgery," International Journal of Medical Robotics and Computer Assisted Surgery, vol. 14, no. 4, Article ID e1908, 2018.

[26] J. Kim, S.-i. Kwon, and K. Kim, "Novel block mechanism for rolling joints in minimally invasive surgery," Mechanism and Machine Theory, vol. 147, Article ID 103774, 2020.

[27] K. Kim, H. Woo, and J. Suh, "Design and evaluation of a continuum robot with discreted link joints for cardiovascular interventions," in Proceedings of the 2018 7th IEEE International Conference on Biomedical Robotics and Biomechatronics (Biorob), IEEE, pp. 627-633, Enschede, Netherlands, Auguest 2018.

[28] R. J. Webster III and B. A. Jones, "Design and kinematic modeling of constant curvature continuum robots: a review," The International Journal of Robotics Research, vol. 29, no. 13, pp. 1661-1683, 2010.

[29] J. Barrientos-Diez, X. Dong, D. Axinte, and J. Kell, "Real-time kinematics of continuum robots: modelling and validation," Robotics and Computer-Integrated Manufacturing, vol. 67, Article ID 102019, 2021.

[30] X. Dong, M. Raffles, S. C. Guzman, D. Axinte, and J. Kell, "Design and analysis of a family of snake arm robots connected by compliant joints," Mechanism and Machine Theory, vol. 77, pp. 73-91, 2014.

[31] L. Tang, J. Wang, Y. Zheng, G. Gu, L. Zhu, and X. Zhu, "Design of a cable-driven hyper-redundant robot with experimental validation," International Journal of Advanced Robotic Systems, vol. 14, no. 5, Article ID 1729881417734458, 2017.

[32] A. Yeshmukhametov, K. Koganezawa, and Y. Yamamoto, "Design and kinematics of cable-driven continuum robot arm with universal joint backbone," in Proceedings of the 2018 IEEE International Conference on Robotics and Biomimetics (ROBIO), IEEE, pp. 2444-2449, Kuala Lumpur, Malaysia, December 2018.

[33] R. Xue, B. Ren, Z. Yan, and Z. Du, “A cable-pulley system modeling based position compensation control for a laparoscope surgical robot," Mechanism and Machine Theory, vol. 118, pp. 283-299, 2017.

[34] J. W. Suh and K. Y. Kim, "Harmonious cable actuation mechanism for soft robot joints using a pair of noncircular pulleys," Journal of Mechanisms and Robotics, vol. 10, no. 6, 2018.
[35] Z. Li and R. Du, "Design and analysis of a bio-inspired wiredriven multi-section flexible robot," International Journal of Advanced Robotic Systems, vol. 10, no. 4, Article ID 209, 2013.

[36] Y. Liu and F. Alambeigi, "Effect of external and internal loads on tension loss of tendon-driven continuum manipulators," IEEE Robotics and Automation Letters, vol. 6, no. 2, pp. 1606-1613, 2021.

[37] S. M. Segreti, M. D. Kutzer, R. J. Murphy, and M. Armand, "Cable length estimation for a compliant surgical manipulator," in Proceedings of the 2012 IEEE International Conference on Robotics and Automation, IEEE, pp. 701-708, Saint Paul, MN, USA, May 2012.

[38] H. In, H. Lee, U. Jeong, B. B. Kang, and K. J. Cho, "Feasibility study of a slack enabling actuator for actuating tendon-driven soft wearable robot without pretension," in Proceedings of the 2015 IEEE International Conference on Robotics and Automation (ICRA), IEEE, pp. 1229-1234, Seattle, WA, USA, May 2015.

[39] B. A. Jones and I. D. Walker, "Kinematics for multisection continuum robots," IEEE Transactions on Robotics, vol. 22, no. 1, pp. 43-55, 2006.

[40] Y. Y. Zhou, J. H. Li, M. Q. Guo, Z. D. Wang, and H. Liu, "Modeling and optimization analysis of a continuum robot for single-port surgery," Robot, vol. 42, no. 3, pp. 316-324, 2020.

[41] G. Palmieri and C. Scoccia, "Motion planning and control of redundant manipulators for dynamical obstacle avoidance," Machines, vol. 9, no. 6, Article ID 121, 2021.

[42] S. Jin, S. K. Lee, J. Lee, and S. Han, "Kinematic model and realtime path generator for a wire-driven surgical robot arm with articulated joint structure," Applied Sciences, vol. 9, no. 19, Article ID 4114, 2019.

[43] T. Kato, I. Okumura, H. Kose, K. Takagi, and N. Hata, "Tendon-driven continuum robot for neuroendoscopy: validation of extended kinematic mapping for hysteresis operation," International Journal of Computer Assisted Radiology and Surgery, vol. 11, no. 4, pp. 589-602, 2016.

[44] M. Hwang and D.-S. Kwon, "Strong continuum manipulator for flexible endoscopic surgery," IEEE, vol. 24, no. 5, pp. 2193-2203, 2019.

[45] H. Yuan, P. W. Y. Chiu, and Z. Li, "Shape-reconstructionbased force sensing method for continuum surgical robots with large deformation," IEEE Robotics and Automation Letters, vol. 2, no. 4, pp. 1972-1979, 2017. 